\title{
Elucidating the innate immunological effects of mild magnetic hyperthermia on U87 human glioblastoma cells: an in vitro study
}

\author{
Stefano Persano ${ }^{1,}{ }^{*}$, Francesco Vicini ${ }^{1}$, Alessandro Poggi ${ }^{2}$, Jordi Leonardo Castrillo Fernandez ${ }^{2}$, Giusy Maria Rita \\ Rizzo $^{1}$, Helena Gavilán ${ }^{1}$, Niccolo Silvestri ${ }^{1}$ and Teresa Pellegrino ${ }^{1, *}$ \\ ${ }^{1}$ Nanomaterials for Biomedical Applications Department, Istituto Italiano di Tecnologia (IIT), via Morego 30, \\ 16163 Genoa, Italy. \\ ${ }^{2}$ Molecular Oncology and Angiogenesis Unit, IRCCS Ospedale Policlinico San Martino, 16132 Genoa, Italy. \\ *Correspondence: Co-corresponding authors: e-mail: stefano.persano@iit.it; e-mail: teresa.pellegrino@iit.it
}

Citation: Lastname, F.; Lastname, F.; Last-name, F. Title. Pharmaceutics 2021, 13, $x$.

https://doi.org/10.3390/xxxxx

Received: date

Accepted: date

Published: date

Publisher's Note: MDPI stays neutral with regard to jurisdictional

claims in published maps and institutional affiliations.

Copyright: (c) 2020 by the authors. Submitted for possible open access publication under the terms and conditions of the Creative Commons Attribution (CC BY) license (http://creativecommons.org/licenses/by/4.0/).
Abstract: Cancer immunotherapies have been approved as standard second-line or in some cases even as first-line treatment for a wide range of cancers. However, immunotherapy has not shown a clinically relevant success in glioblastoma (GBM). This is principally due to the brain's "immune-privileged" status and the peculiar tumor microenvironment (TME) of GBM characterized by a lack of tumor-infiltrating lymphocytes and the establishment of immunosuppressive mechanisms.

Herein, we explored local mild thermal treatment, generated by cubic-shaped iron oxide magnetic nanoparticles (size $\sim 17 \mathrm{~nm}$ ) when exposed to an external alternating magnetic field (AMF), to induce immunogenic cell death (ICD) in U87 glioblastoma cells. In accordance with what has been observed with other tumor types, we found that mild magnetic hyperthermia modulates the immunological profile of U87 glioblastoma cells by inducing stress-associated signals leading to enhanced phagocytosis and killing of U87 cells by macrophages. At the same time, we demonstrated that mild magnetic hyperthermia has a modulatory effect on the expression of inhibitory and activating NK cell ligands on target cells. Interestingly, alteration in the expression of NK ligands in U87 cells by mild magnetic hyperthermia treatment, increased their susceptibility to NK cell killing and enhanced NK cell functionality. The overall findings demonstrate that mild magnetic hyperthermia stimulates ICD and sensitizes GBM cells to NK-mediated killing by inducing the upregulation of specific stress ligands, providing a novel immunotherapeutic approach for GBM treatment, with potential to synergize with existing NK cell-based therapies thus improving their therapeutic outcomes.

Keywords: Glioblastoma; immunogenic cell death; innate immunity; Natural Killer; macrophages; magnetic hyperthermia

\section{Introduction}

Glioblastoma (GBM) is the most common type of primary malignant brain tumor and is one of the most aggressive and lethal forms of cancer, with a median survival rate of 12-15 months following diagnosis [1]. The current standard treatment protocol, involving maximal surgical resection of the tumor, followed by concomitant administration of radiotherapy and chemotherapy, mostly with temozolomide (TMZ), has not produced a satisfactory life-extension, and indeed, less than $5 \%$ of patients diagnosed with GBM survive for more than 5 years [2].

In the last decade, immunotherapy has emerged as a promising therapeutic regimen for cancer therapy, showing great success in the treatment of numerous cancers, including melanoma, lung, breast, colorectal and kidney cancer, and a number of immunotherapy 
protocols have been approved by the Food and Drug Administration (FDA) for clinical use [3-9]. Despite the encouraging results achieved by cancer immunotherapy in many lymphomas and solid tumors, the outcomes in GBM patients have been rather disappointing [10]. The establishment of an immunosuppressive tumor microenvironment (TME) and the presence of the blood-brain barrier (BBB), rendering GBM an "immune-privileged" site, have been widely recognized as the main cause of this failure [11-13].

Brain tumor cells can directly inhibit immune responses by regulating the expression of specific surface proteins, including programmed death-ligand 1 (PD-L1), cluster of differentiation 47 (CD47), human leukocyte antigen (HLA) molecules and Natural killer group 2 member D (NKG2D) ligands; or indirectly through the secretion of anti-inflammatory cytokines or chemokines promoting the recruitment of immunosuppressive cells like tumor-associated macrophages (TAM) and myeloid-derived suppressor cells (MDSC), which constitute up to $50 \%$ of the glioma mass. [14].

Most cancer immunotherapy treatments have focused on unleashing antigen-specific immune responses, driven mostly by $\mathrm{CD}^{+} \mathrm{T}$ cells[4]. The key role played by effector $\mathrm{T}$ cells in mediating antitumor responses has been corroborated by the positive correlation existing between tumor infiltrating $T$ cells and prognostic outcomes [15-17]. However, it has been demonstrated that the onset and maintenance of $\mathrm{CD} 8^{+} \mathrm{T}$ cell-mediated responses and the generation of long-term immunity against tumors relies on the efficient activation of the innate immune system [18-20]. Additionally, innate immune cells not only sense cancer cells and initiate adaptive immune responses, but they can also exert an effector response through specific mechanisms, such as phagocytosis for macrophages and natural cytotoxicity for natural killer (NK) cells [21,22]. The enormous potential of innate immunity in GBM has been underlined by multiple studies showing that NK cells represent the most abundant intratumoral lymphocyte population, and that GBM cells may be particularly vulnerable to innate effector lymphocytes [23-28]. The relevant role of innate immunity in cancer therapy is further supported by recent findings that have confirmed the existence of a subpopulation of NK cells which exhibits antigen or non-antigen specific memory-like functions, revealing that NK cells can possess features analogous to adaptive immunity [29-32].

The recognition of cancer cells by effector innate immune cells occurs via specific receptors like NKG2D, which can detect molecular alterations, such as cell surface expression of stress-inducible molecules (e.g. MHC class I chain-related proteins A and B (MICA/B) and UL16 binding proteins (ULBPs)) on target cells [33]. Innate immune cells also participate to effector responses via antibody-dependent cellular phagocytosis (ADCP) or antibody-dependent cellular cytotoxicity (ADCC) [34,35].

The importance of innate immunity is turning out to be particularly relevant in those tumors, such as GBM, that are characterized by low mutation burden, downregulation or loss of the antigen presentation, $\mathrm{T}$ cell dysfunction and low number of tumor-infiltrating $\mathrm{T}$ cells, which can limit the utility of immunotherapeutic strategies directed to promote an antigen-specific $T$ cell response (e.g. anticancer vaccines) [13,36].

In this context, therapeutic approaches aiming to sensitize GBM cells to the recognition and killing by innate immune cells represent an effective strategy to improve the outcomes of immunotherapy.

Immunogenic cell death (ICD)-inducing strategies are a convenient mode to achieve simultaneous activation of innate and adaptive immunity by promoting the expression and exposition of stress-associated molecules and the release of tumor antigens normally hidden within cancer cells [37,38]. ICD is accompanied by the exposure and release of numerous damaged-associated molecular patterns (DAMPs), including calreticulin (CTR), heat shock proteins (HSPs) and high mobility group box 1 (HMGB1). CRT exposed on the surface of dying cancer cells promotes the engulfment of dying cancer cells by antigen-presenting cells (APCs), whereas HSPs and HMGB1 bind to TLR4, enhancing APC activation and antigen cross-presentation [37-39]. Ultimately, ICD serves as a source of 
both antigens and adjuvanting molecules to activate APCs and promote antitumor immunity, providing an opportunity for a novel treatment known as "in situ vaccination" [40-42].

Different treatments have been identified as ICD inducers, including chemotherapy, radiotherapy and hyperthermia (HT) [43-48]. Among all, HT presents unique advantages over other ICD inducers, such as minimal invasiveness, inferior mutagenic potential and systemic toxicity, and improved tumor specificity $[49,50]$. All this is particularly true when heat is selective and locally applied at the tumor site sparing the surrounding healthy tissues or other organs. This is the case of magnetic hyperthermia (MHT) which relies on the use of magnetic nanoparticles to remotely induce local heat when an alternating magnetic field (AMF) is externally applied [51,52]. Moreover, in comparison to conventional HT treatments, coupling plasmonic nanoparticles with laser light as external energy source, MHT exhibits a superior penetration with no tissue-depth attenuation of the magnetic responsiveness of magnetic nanoparticles under magnetic field conditions $(100 \mathrm{kHz}$ and $24 \mathrm{kA} / \mathrm{m}$ ) that are clinically safe for patients, rendering MHT suitable for effective heating of deep-seated tumors [53].

Although MHT has been largely investigated as ICD-inducer, the focus of these studies has been mainly toward its ability to prime a cytotoxic $\mathrm{T}$ lymphocyte (CTL)-dependent immune response against cancer cells, while the role played by innate effector cells in mediating ICD-induced responses has not been fully characterized [48,53-55]. Indeed, ICD-associated DAMPs not only favor the recruitment and activation of APCs but many of them, including HSPs, HMGB1, NKG2D ligands, have been also demonstrated to engage receptors expressed on NK cells, potentially sensitizing tumor cells to lysis by NK cells $[56,57]$. Prior reports support that when locally applied, MHT can also have a direct effect on tumor-resident immune cells by promoting a decrease of MDSCs and TAM repolarization from M2 to M1 phenotype [48,59]. Upon activation, macrophages and NK cells can not only initiate and shape adaptive immune responses and function as cytokine producers, but they can also act as effector cells and be directly responsible for tumor cell elimination [59].

Here, the capacity of mild MHT $\left(43^{\circ} \mathrm{C}\right)$ to induce ICD, and thereby increase GBM cells' susceptibility to innate effector cells, was evaluated on U87 GBM cells grown as 3D spheroids (Figure 1a and $\mathbf{1 b}$ ). The study was conducted using iron oxide nanocubes (IONCs) with a size of approximately $17 \pm 2 \mathrm{~nm}$ and functionalized with gallic acid (GA)Polyethylene glycol (PEG) polymer to improve the colloidal stability of the resulting IONC-GA-PEG, thus preserving their heating properties in biological environments.

We believe that this work offers a new rationale and design considerations for the development of novel nanotechnology-based treatments directed at eliciting both innate and adaptive immunity, for a more robust an effective antitumor immune response. 


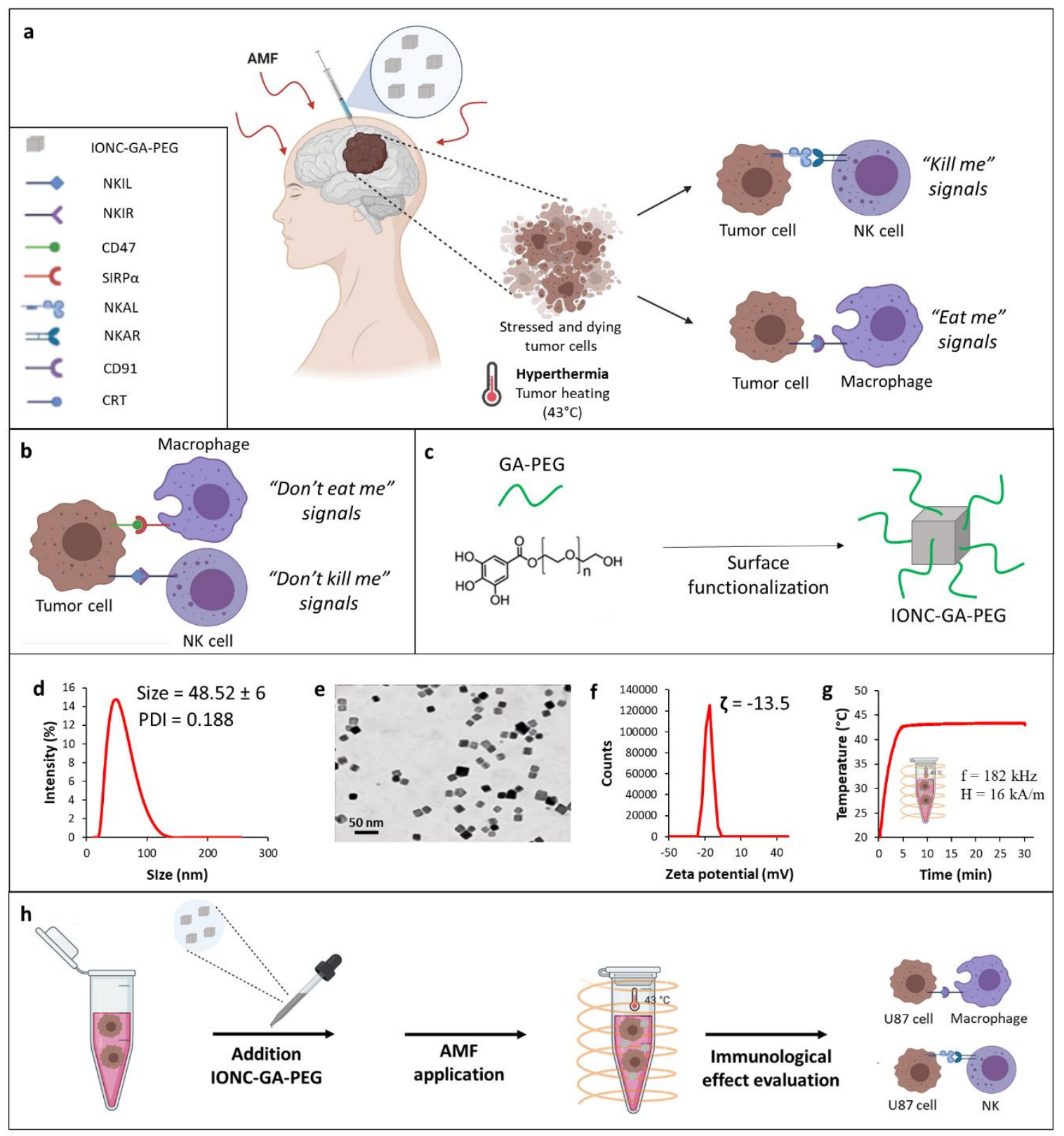

Figure 1. Schematic representation of the innate immunological effect of mild MHT $\left(43^{\circ} \mathrm{C}\right)$ on tumor cells (a). Tumor cells express on their surface anti-phagocytic molecules ("don't eat me signal"), such as CD47, that binding to SIRP- $\alpha$ receptor on macrophages impedes macrophage phagocytosis of tumor cells (b). Tumor cells can also evade NK cell-mediated killing by increasing the expression of NK inhibitory ligands (NKILs) recognized by specific receptors (NKIRs) on NK cells, and downregulating the expression of NK activating ligands (NKALs) responsible for inducing the activation of a receptor-mediated NK killing of tumor cells by engaging specific NK activating receptors (NKARs) on NK cells (b). All this results in a "don't kill me" signal that allows evasion of NK cell immune responses. Mild MHT can revert the TIME thus rendering tumor cells more susceptible to innate immune effectors (macrophages and NK cells) by enhancing the expression of ICD-associated markers, like CRT engaging CD91 receptor on macrophages, and concomitantly upregulating NKALs, and downregulating NKILs (a). Illustration of the functionalization of IONCs with GAPEG polymer (c). Characterization of hydrodynamic size and PDI of IONC-GA-PEG by dynamic light scattering (DLS) (d). Representative TEM image of the IONC core coated by GA-PEG polymer (IONC-GA-PEG) (e). Zeta potential of IONC-GA-PEG (f). Temperature vs time heat profile of IONC-GA-PEG to heat up to the desired temperature of $43^{\circ} \mathrm{C}$ under an AMF at frequency, $f$, of 182 $\mathrm{kHz}$ and field intensity, $\mathrm{H}$, of $16 \mathrm{kA} / \mathrm{m}(\mathrm{g})$. A schematic illustration of the step-by-step in vitro study $(\mathrm{h})$.

\section{Results}

\subsection{Synthesis and characterization of GA-PEG-coated IONCS}

The synthesis of highly monodispersed IONCs with a size of $17 \pm 2 \mathrm{~nm}$ coated by surfactant molecules (oleic acid) was conducted according to the patented procedure [60]. Gallic polyethylene glycol $\left(\mathrm{Mr}_{\mathrm{r}}\right.$ 1,500) (GA-PEG) was chosen as a 
water transfer ligand for the IONCs (Figure 1c). We adapted a well-established protocol from our lab for the synthesis of GA-PEG and its further use for the water transfer of the IONCs, achieving the desired well-soluble IONC-GA-PEG in aqueous media [61].

The success of the water transfer was also indicated by the mono-modal size distribution of the hydrodynamic peak as measured by dynamic light scattering (DLS) spectra, which was centered at $48 \pm 6 \mathrm{~nm}$ and had a polydispersity index (PDI) of 0.188 (Figure 1d). The synthesized IONCs-GA-PEG were further characterized by transmission electron microscopy (TEM) analysis, which confirmed the presence monodispersed nanocubes separated from each other and without the presence of aggregates (Figure 1e and S1 in the supporting information). The overall charge of IONCs grafted with GA-PEG molecules determined by zeta potential was slightly negative $(-13.5 \mathrm{mV})$ (Figure 1f).

The heating properties of IONCs were measured by calorimetric measurements when exposing the colloidal solution of IONCs $\left(2 \mathrm{~g}_{[\mathrm{Fe}]} / \mathrm{L}\right)$ to an external AMF. SAR values of the PEGylated IONCs were measured at different conditions (frequencies: 100 or 300 $\mathrm{kHz}$; amplitudes: 12,16 or $24 \mathrm{kA} / \mathrm{m}$ ), confirming its high SAR values at different frequency and field amplitude of safety clinical use (Figure S2 in the supporting information).

It is relevant to highlight that PEGylated IONCs were able to increase the temperature media at a target therapeutic temperature of $43^{\circ} \mathrm{C}$ within the initial 5 minutes, at a low IONC dose $\left(2 \mathrm{~g}_{[\mathrm{Fe} /} / \mathrm{L}\right)$ and under field conditions considered clinically safe (magnetic field intensity, $\mathrm{H}$, of $16 \mathrm{kA} / \mathrm{m}$ and at a frequency, $f$, of $182 \mathrm{kHz}$ ) (Figure 1g).

To evaluate the biocompatibility of PEG-functionalized IONCs, an in vitro viability assay using the cell counting kit-8 (CCK-8) was conducted on human U87 glioblastoma cells (Figure S3 in the supporting information). Control groups that received either no treatment or that were exposed to AMF or IONCs alone did not show any significant variation of cell viability at 24 and 48 hours. A cytotoxicity effect was observed only when U87 cells incubated with IONCs were subjected to three cycles of AMF, showing a reduction of cell viability up to more than 60\% (Figure S3 in the supporting information). Taken together, these results clearly indicate that GA-PEG-capped IONCs possess optimal colloidal stability, high heat transfer efficiency and low toxicity profile in absence of MHT actuation. Thus, IONC-GA-PEG represents an excellent heating mediator for the development of magnetic nanoparticle-mediated hyperthermia therapies.

Next, these nanoparticles were used for the in vitro studies with U87 GBM cells to evaluate the immunological effect of mild MHT. A schematic of the overall study performed in U87 cells is shown in Figure 1h.

\subsection{Mild MHT induces apoptosis and impacts tumor-initiating and migratory abilities of U87} cells

Mild hyperthermia has been shown to be effective at inducing ICD in many types of cancer cells, including glioblastoma cells $[48,53,54,62]$. ICD is a form of cell death characterized by the exposition and/or release of various DAMPs, which stimulate immune cell recruitment and activation by engaging specific receptors on the surface of innate immune cells [37]. Cell death can occur through numerous regulated mechanisms defined by specific molecular events and physiological effects. Secondary necrosis, in contrast to apoptosis or primary necrosis, is considered to be more immunogenic, as it can initiate proinflammatory processes leading to the activation of innate and adaptive immune responses [63]. 
a

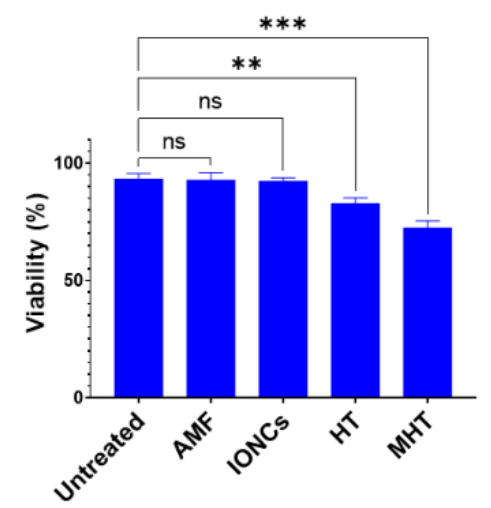

b

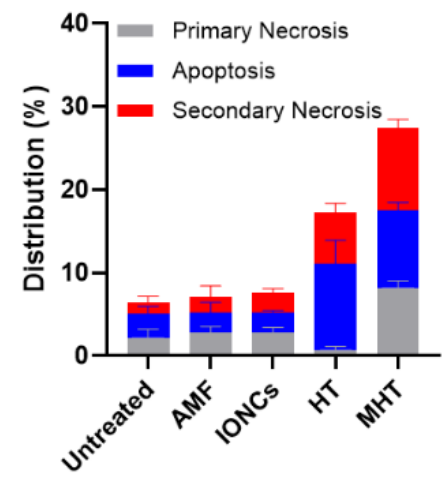

C

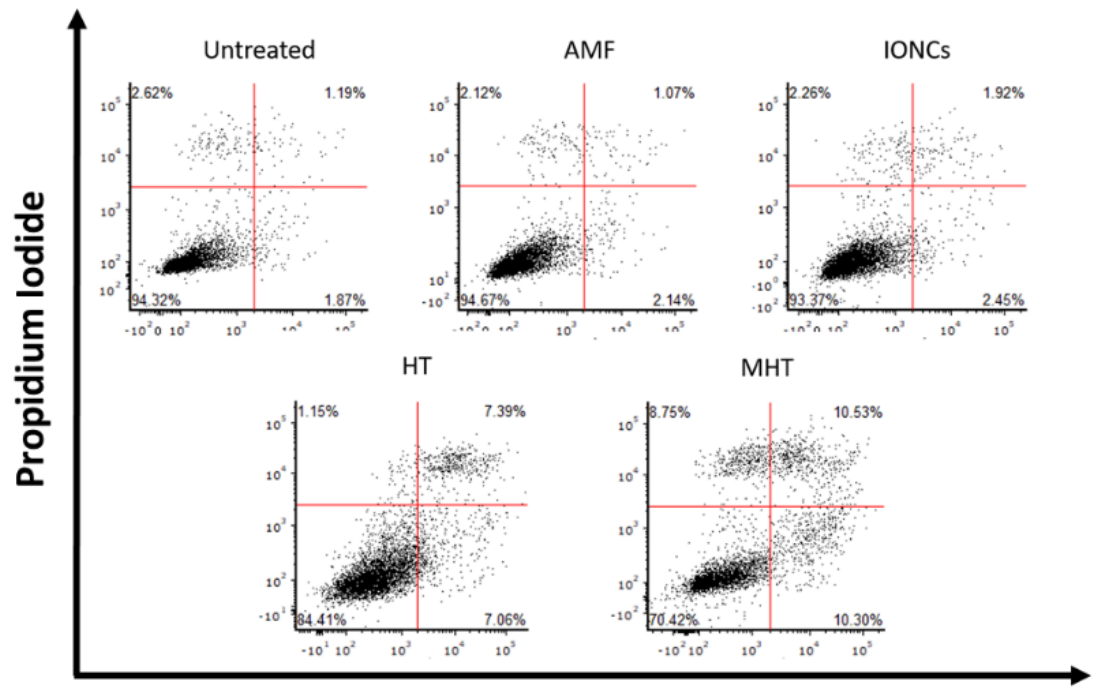

Annexin-FITC

Figure 2. Evaluation of the ability of mild MHT to induce cell death in U87 cells measured by Annexin V/PI assay. MHT induces reduction of cell viability in U87 (a). After being exposed to mild MHT ( 3 cycles of $30 \mathrm{~min}$ at $43^{\circ} \mathrm{C}$ ), U87 cells were left to rest for $24 \mathrm{~h}$ and then stained with annexinV-FITC and PI. Frequency of apoptotic (Annexin $\mathrm{V}^{+} / \mathrm{PI}^{-}$) (blue bars), primary necrotic (Annexin $\mathrm{V}^{-}$ $/ \mathrm{PI}^{+}$) (grey bars) and secondary necrotic (Annexin $\mathrm{V}^{+} / \mathrm{PI}^{+}$) (red bars), U87 cells were determined by flow cytometry $(b, c)$. Data are represented as mean \pm SD of three independent experiments $(n=3)$ (b). Representative dot plots of Annexin V/PI staining of U87 cells treated with AMF, IONCs, HT or MHT(c). Statistical significance was determined with a two-tail unpaired student's test $\left({ }^{*} 0.01<\mathrm{p}<\right.$ $0.05 ;{ }^{* *} 0.001<\mathrm{p}<0.01 ;{ }^{* * *} \mathrm{p}<0.001 ; \mathrm{n} . \mathrm{s} .=$ not significant $)$.

In order to ascertain the ability of MHT to evoke ICD in GBM, U87 spheroids were treated with mild $\mathrm{MHT}\left(43^{\circ} \mathrm{C}\right)$ and subsequently incubated at $37^{\circ} \mathrm{C}$ for $24 \mathrm{~h}$ in serum-free medium. Cell viability in cancer cells was then evaluated by Annexin V/PI staining followed by flow cytometric analysis (Figure 2). The results showed that MHT treatment caused a reduction in cell viability of approximately $30 \%$ in U87 cells at 24 hours posttreatment (Figure 2a). Interestingly, conversely to what was observed in tumor cells treated with classical hyperthermia (HT), carried out at the constant temperature of $43^{\circ} \mathrm{C}$ using a thermomixer, a higher number of Annexin V/PI double positive cells (secondary necrosis) were detected at 24 hours after treatment with MHT (Figure $\mathbf{2 b}$ and $\mathbf{2 c}$ ). The cytotoxic effect of MHT in U87 cells was also confirmed with a colony-forming assay (Figure 3a) and further characterized by flow cytometric analysis of the cell cycle (Figure S4, supporting information). We found that mild MHT strongly inhibits cell proliferation in U87 cells as a consequence of an arrest of the cell cycle in the G2/M phase as evinced by the accumulation of cells in S and G2/M phase (Figure S4 in the supporting information). 


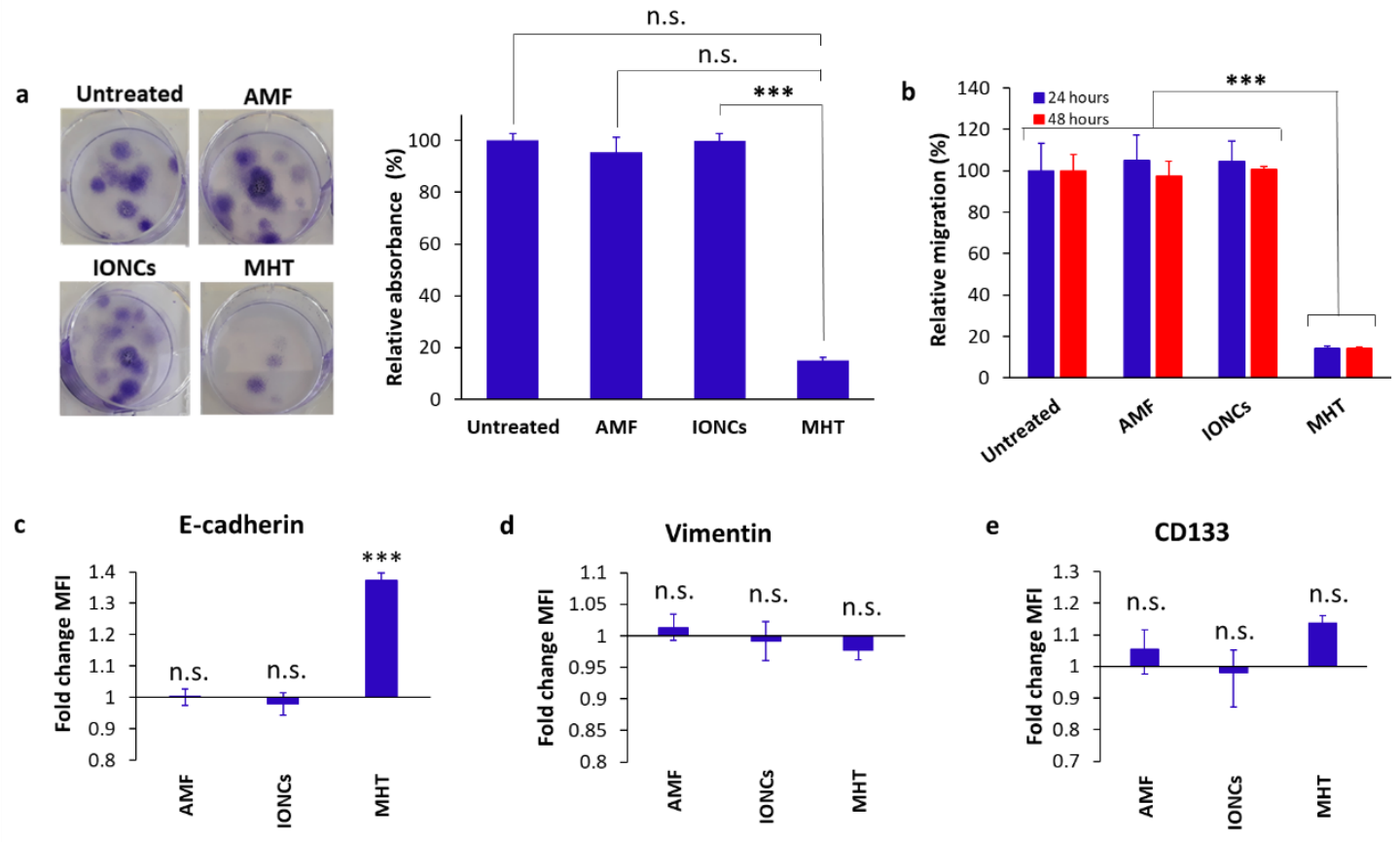

Figure 3. Evaluation of the clonogenic (a) and migration (b) activity, surface expression of EMT (c, d) and CSC markers (e), in U87 cells in response to the treatment with IONCs and mild MHT (3 cycles of 30 minutes at $43^{\circ} \mathrm{C}$ ). Clonogenicity was evaluated in U87 cells after treatment with AMF, IONCs and MHT (a). Data are shown as representative images of stained colony with crystal violet and relative absorbance at $595 \mathrm{~nm}$. A transwell assay was used to determine the migration and invasion ability of U87 cells after exposition to different treatments such as AMF only (AMF), IONCs only (IONCs) and IONCs + AMF (MHT) (b). The results were represented as relative percentage compared to untreated cells (d). Error bars indicate \pm SD calculated from three independent experiments $(n=3)$. Surface expression of E-cadherin, Vimentin and CD133 was measured by flow cytometry at 24 hours post-treatment with AMF, IONCs and MHT, and data reported relative to untreated controls $(c, d, e)$. Data are represented as mean \pm SD of three independent experiments $(n=3)$. Statistical analysis was conducted with a two-tail unpaired student's test $\left({ }^{*} 0.01<\mathrm{p}<0.05 ;{ }^{* *} 0.001<\mathrm{p}<\right.$ $0.01 ;{ }^{* * *} \mathrm{p}<0.001 ; \mathrm{n} . \mathrm{s} .=$ not significant $)$.

Tumor cell migration and epithelial-mesenchymal transition (EMT) are key mechanisms that facilitate tumor progression by supporting metastasis formation, cancer stem cell (CSC) generation and drug resistance [64].

A transwell assay was set up to evaluate the effect of mild MHT on cell migration. Our findings showed that mild MHT strongly suppressed U87 tumor cell migration (up to ten-fold) at 24- and 48-hours post-treatment (Figure 3b). Flow cytometric analysis of MHT-treated cells revealed that this observation was accompanied by increased ectopic levels of E-cadherin (Figure 3c), whereas no remarkable changes were found in the surface expression level of both CD133 and vimentin in response to treatment with MHT (Figure 3d and 3e). The increment in the levels of E-cadherin found in MHT-treated cells are in line with the reduced migration ability seen in U87 cells after treatment with mild MHT, since cancer cells with an epithelial phenotype are commonly characterized by a lower migration rate than those with a mesenchymal phenotype.

\subsection{Mild MHT induces ICD in U87 cells and enhances macrophages' antitumoral functions.}

Cells undergoing apoptosis exhibit changes involving the exposure and release of DAMPs, such as calreticulin (CRT), HSPs, HMGB1 and other molecules, which act as danger signals to induce ICD that evoke systemic antitumor immunity. 
To investigate whether MHT can act as ICD-inducer in U87 cells, MHT-treated cancer cells were analyzed for the expression and secretion of ICD-associated molecules. After treatment, we observed an increase in HMGB1 secretion (Figure S5a, supporting information), as well as in the expression of the endoplasmic reticulum (ER) chaperone protein, CRT, and the cytoplasmic chaperone proteins HSP70 and HSP90 on the surface of U87 cells grown in 3D conditions (Figure 4).
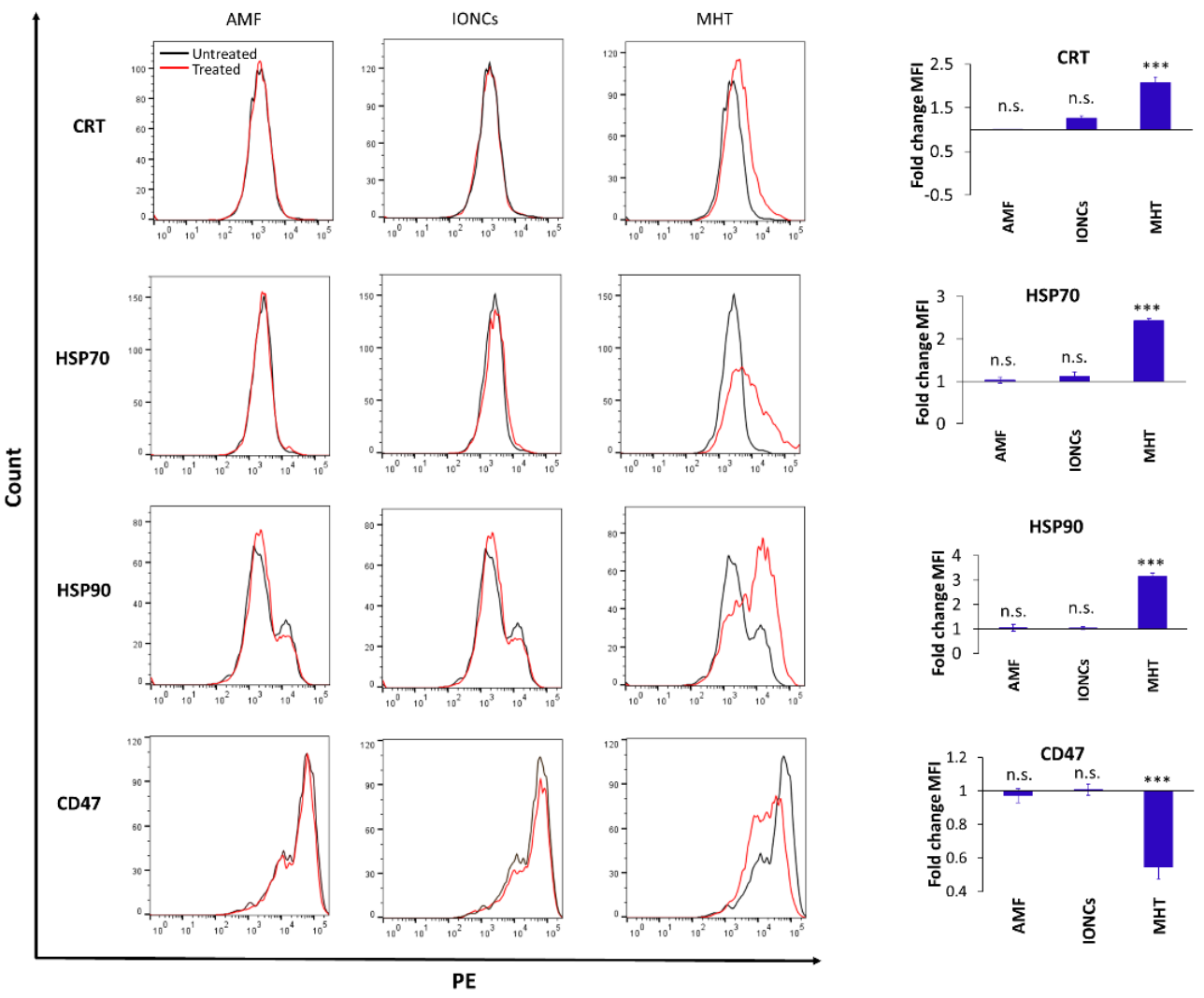

Figure 4. Flow cytometric evaluation of changes in surface expression of ICD-associated markers at 24 hours post-treatment: CRT, HSP70, HSP90 and CD47 on U87 cells exposed to different treatments (AMF, IONCs and MHT) over untreated cells. Data are presented as representative histograms and average fold change (mean \pm SEM) of the mean fluorescence intensity (MFI) of three independent experiments $(n=3)$. Statistical analysis was conducted via two-tailed unpaired student's $t$ test ${ }^{*} 0.01$ $<\mathrm{p}<0.05 ;{ }^{* *} 0.001<\mathrm{p}<0.01 ;{ }^{* * *} \mathrm{p}<0.001 ;$ n.s.= not significant).

Similarly, the expression of CD47 was downregulated in MHT-treated cells compared to the control groups (Figure 4). In all cases, the expression was assessed by flow cytometry $24 \mathrm{~h}$ after exposure to the treatments in cells cultured as 3D spheroids.

These changes in the expression of DAMPs, induced by treatment with MHT, can render cancer cells recognizable to macrophages through both downregulation of "don't eat me" (CD47) and upregulation of "eat me" CRT signals. Thus, we evaluated in vitro the phagocytosis of MHT-treated U87 cells by THP-1 macrophages (Figure 5a and 5b). U87 cells exposed to mild MHT showed higher engulfment by macrophages, compared to untreated cells (Figure 5b). Additionally, in agreement with previously published studies, phagocytosis of MHT-treated U87 cells by macrophages was further enhanced in the presence of the toll-like receptor 9 (TLR9)-agonist cytosine-phosphorothioate-guanine oligodeoxynucleotide (CpG-ODN, $1 \mu \mathrm{g} / \mathrm{mL}$ ).

We further evaluated the ability of U87 cells undergoing ICD in response to mild MHT to induce the activation of macrophages. Consistent with MHT-induced expression of DAMPs, co-culture of THP-1-derived macrophages with MHT-treated U87 cells resulted in the upregulation of CD86 and HLA-DR (Figure 5d), both markers of APC maturation. Finally, we also demonstrated that pretreatment with mild MHT sensitizes U87 GBM cells to the killing mediated by macrophages (Figure 5c). Indeed, the viability of 
MHT-pretreated U87 cells was further reduced when they were co-cultured with macrophages ( $p<0.001)$, while only a modest macrophage-mediated killing was observed when THP-1 macrophages were co-cultured with untreated control cells or cancer cells exposed only to IONCs (Figure 5c). Together these in vitro results indicate that treatment with MHT can sensitize U87 glioblastoma cells to the killing mediated by macrophages, besides promoting their activation.

.

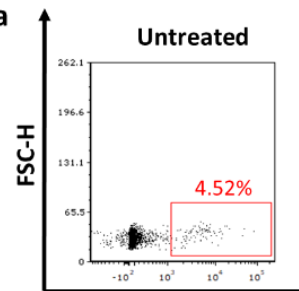

CpG

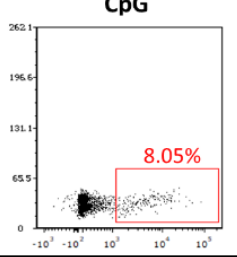

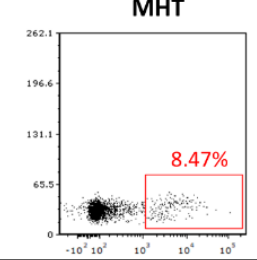

CD11 $\mathrm{b}^{+} \mathrm{CSFE}^{+}$

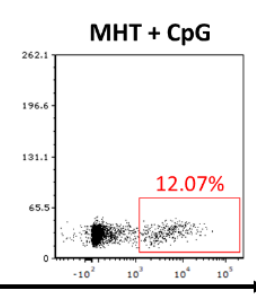

b

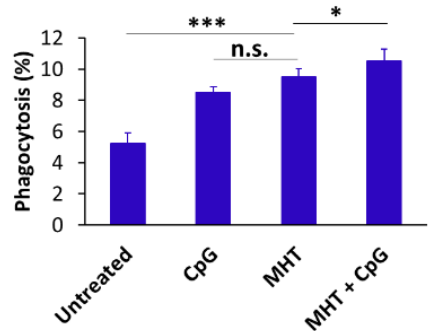

C

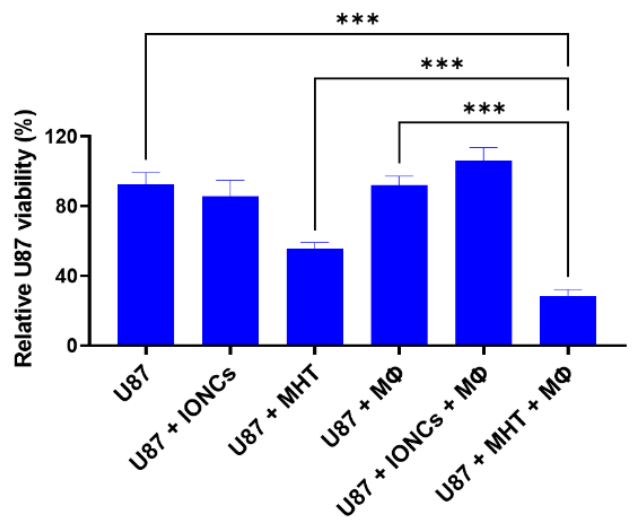

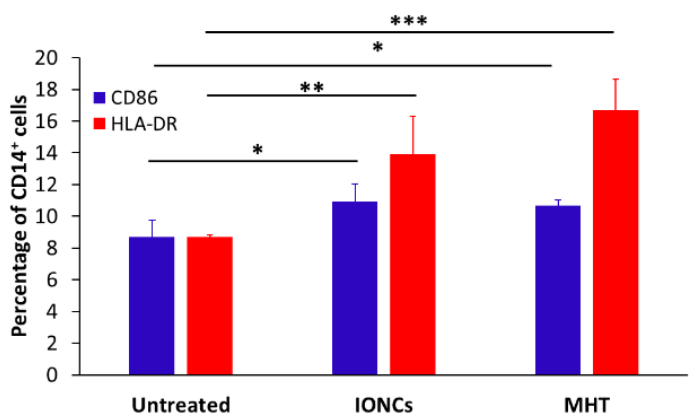

Figure 5. Representative dot plots showing phagocytosis of MHT-treated U87 cells by THP-1-derived macrophages $(\mathrm{M} \phi)$ after 2 hours of coculture at a 1:1 effector: target (E:T) ratio (a). Flow cytometric quantification of phagocytosis rates by THP-1-derived M $\phi$ towards untreated or MHTtreated U87 in the presence or absence of TLR9 agonist CpG (b). Percentage of phagocytosis was determined by the percentage of $\mathrm{CSFE}^{+}$cells within $\mathrm{CD}_{11 \mathrm{~b}^{+} \mathrm{M} \phi}$ cell gate (double positive). Tumor cell killing by macrophages was evaluated by crystal violet staining after 24 hours of co-culture with untreated or treated U87 cells with IONCs only (U87+IONCs) or with IONCs and MHT (U87+MHT) (c). Activation of THP-1-derived macrophages was evaluated by analysis of surface expression of CD86 (blue bars) and HLA-DR (red bars) after 48 of co-incubation with untreated U87 cells, U87 cells exposed to IONCs (IONCs) and MHT-treated U87 cells (MHT) (d). All data are shown as mean \pm SD. Statistical analysis was conducted via two-tailed unpaired student's $t$ test $\left({ }^{*} 0.01<\mathrm{p}<0.05\right.$; ${ }^{* *} 0.001<\mathrm{p}<0.01 ;{ }^{* * *} \mathrm{p}<0.001 ;$ n.s. $=$ not significant $)$.

The PD-1/PD-L1 axis represents another immune checkpoint pathway harnessed by malignant cells to evade antitumor immune responses [65]. While initially it was thought that its role was only restricted to T cells, recent evidences pointed out that PD-1/PD-L1 signaling pathway also participates in negatively controlling innate immune effectors $[65,66]$. Given the importance of PD-L1 in tumor immune evasion, we decided to assess the effect of mild MHT on PD-L1 expression in U87 cells by flow cytometry (Figure S3b and S3c in the supporting information). We found that mild MHT modestly downregulated PD-L1 expression on the surface of GBM cells at 24 hours after treatment, whereas the exposition to AMF or IONCs did not produced any significant changes in the surface levels of PD-L1 (Figure S5b in the supporting information).

\subsection{Mild MHT alters the expression of NK cell-activating and inhibitory ligands on U87 cells}

In line with previously published works, we showed that MHT triggers the upregulation of DAMPs. Despite the role of MHT at inducing DAMPs has been already explored, its effect in modulating the expression of some stress-induced ligands and inhibitory NKligands on GBM cells remains to be characterized. Therefore, we investigated whether 
mild MHT could modulate the expression of NK cell ligands such as MICA, ULBP-1, ULBP-2 (NKG2D ligands); Nectin-2, PVR (DNAM-1 ligands); B7H6 (NKp30 ligand); HLA class I (HLA-I or HLA-ABC) and HLA-E on GBM cells. Flow cytometric analysis revealed an increase in the expression of NKp30 and DNAM-1 ligands B7H6, Nectin-2 and PVR (Figure 6) and a slight reduction in the levels of NKG2D ligands (MICA and ULBP-2) (Figure S6 in the supporting information) in response to MHT treatment. Whereas not appreciable difference was observed in the expression of ULBP-1 between the different groups ( $p>0.05)$ (Figure $\mathbf{S 6}$ in the supporting information).
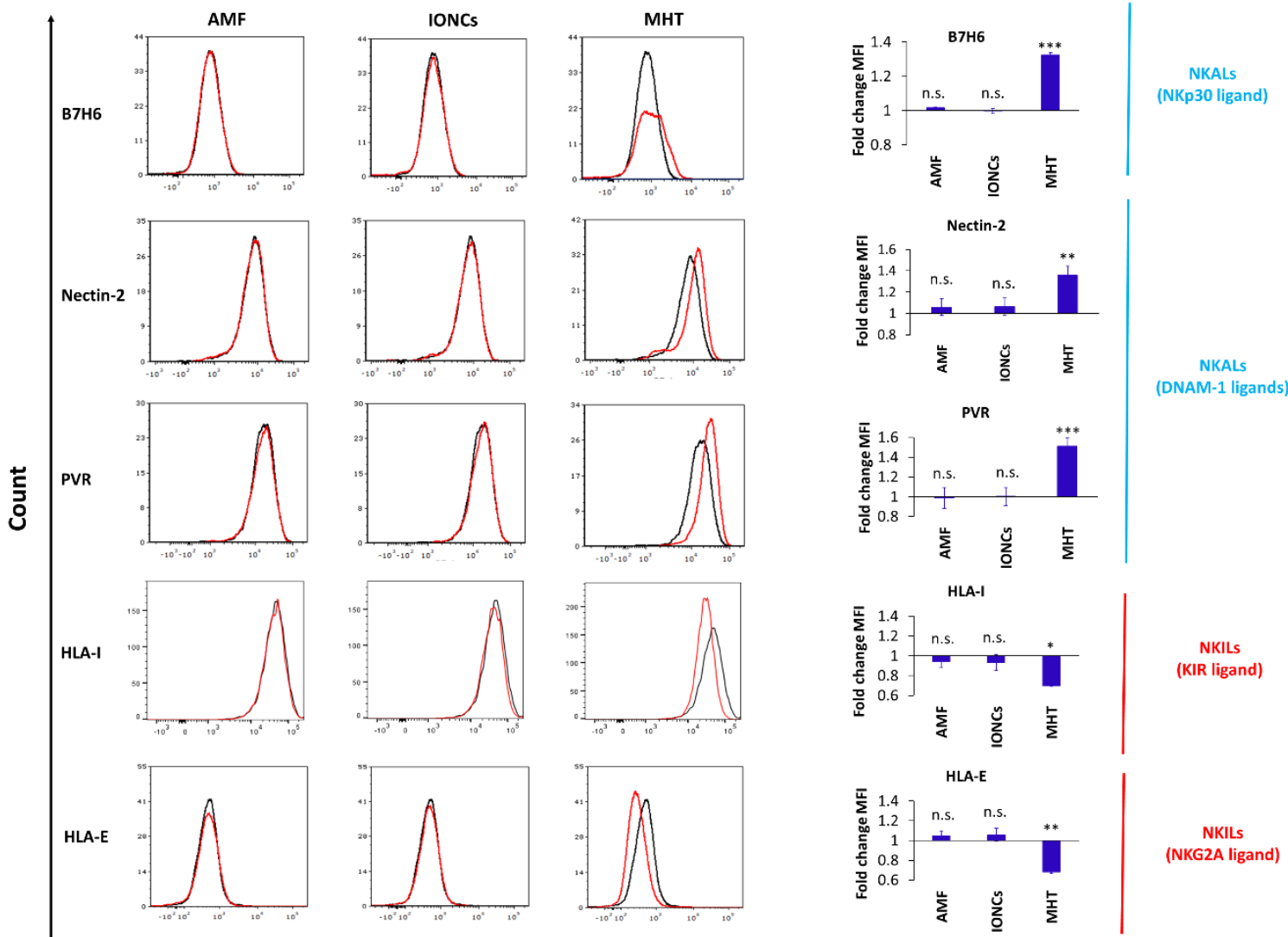

PE $\mathrm{p}<0.01 ;{ }^{* * *} \mathrm{p}<0.001 ;$ n.s. $=$ not significant).

We additionally found that treatment with mild MHT markedly altered the expression of NK cell inhibitory ligands in U87 cells, including both classical (HLA-I) and nonclassical (HLA-E) HLAs (Figure 6). Importantly, for all the examined markers, a non-significant variation in the expression levels was observed in tumor cells groups exposed to AMF or IONCs alone, demonstrating that this re-modulation of NK cell ligands expression is strictly attributable to MHT treatment.

\subsection{Mild MHT sensitizes U87 cells to NK cell-mediated functions.}

Previous studies have reported that GBM stem cells exhibit increased expression of Nectin-2, PVR and B7H6 ligands [26,64]. The first two ligands are recognized by NK cells through DNAM-1 receptor, while B7H6 can promote NK cell activation by interacting with the NKp30 receptor. Overall, the increased expression of these ligands on GBM stem 
a
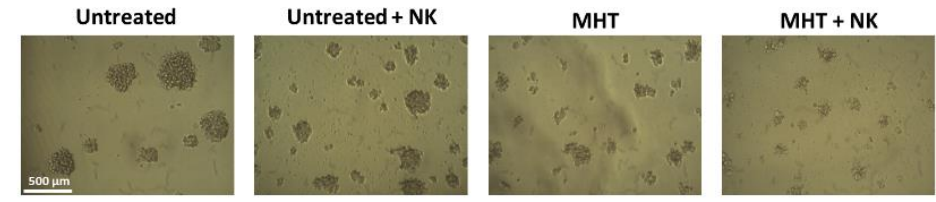

b
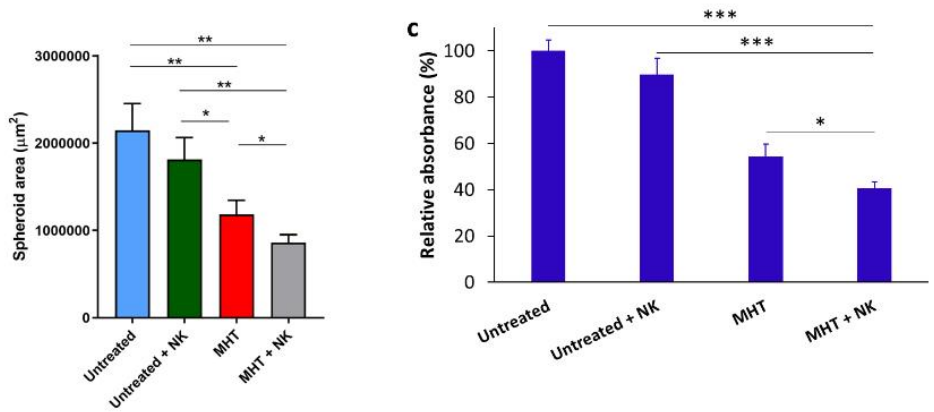

cells has been reported to induce superior receptor-specific activation of NK cells and consequently increase their susceptibility to NK cell-mediated killing [26].

We showed that the expression of CSC-associated ligands can be further upregulated consequently to MHT exposition, and given that their expression has been correlated to increased susceptibility to NK cell-mediated killing, the NK cells killing activity against MHT-treated U87 spheroids was examined. For our purpose U87 spheroids, after MHT treatment, were left to rest for 24 hours in serum-free neural stem cell medium. Next, IL2-activated NK cells were added to pre-treated tumor spheroids, and spheroids growth was monitored. Size analysis of U87 spheroids demonstrated that the combination of MHT and NK cells led to a stronger inhibition of spheroid growth than single treatments (with AMF and no IONCs or with IONCs and no MHT) or NK cells alone (Figure 7a and 7b). The concomitant analysis of cell viability using a crystal violet staining assay (Figure 7c) provided a further evaluation of the effect of MHT as monotherapy or in combination with NK cells on U87 spheroids. Crystal violet staining assay was carried out after 48 hours incubation of harvested cells at conventional adherent conditions, thus allowing alive cancer cells to adhere to the surface of the plate prior to staining. Non-adherent cells (dying U87 cells and NK cells) were removed following extensive washing, and viability was determined by measuring the absorbance of crystal violet using a spectrophotometer. As shown in Figure 7c, a strong reduction in the cell viability was detected in U87 spheroids treated with MHT, indicating that most of the U87 cells had been killed, and this effect was enhanced by NK cells. Indeed, a significant reduction of U87 viability was found in the dual treatment (MHT + NK cells) compared to single treatment with NK cells $(\mathrm{p}<0.001)$ or MHT $(\mathrm{p}<0.001)$, thus indicating that MHT treatment can sensitize U87 GBM cells to NK cell-mediated killing.

Finally, we investigated if NK cell functionality, in terms of migration and cytotoxicity against U87 cells can be improved by the pre-exposure of tumors cells to mild MHT. By performing a transwell migration assay, we found that conditioned media from MHTtreated U87 cells, enhanced the directional movement of NK cells (Figure 7d). Thus providing in vitro evidence that thermal magnetic-triggered therapy could potentially favor NK cell recruitment at the tumor site.
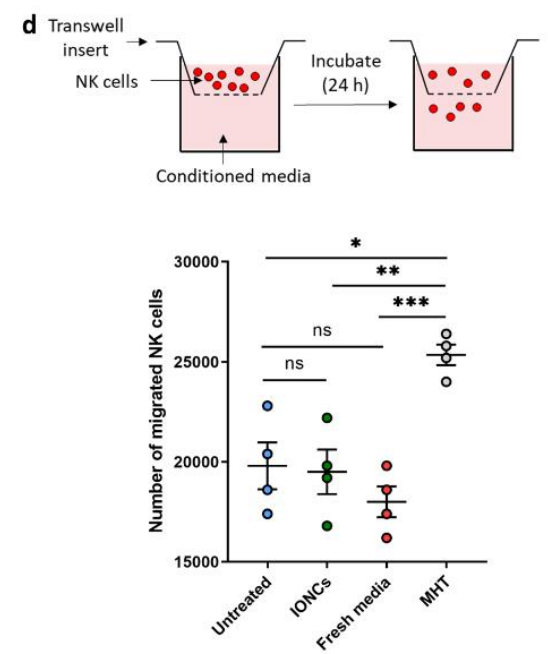

Figure 7. Evaluation of the susceptibility of MHT-treated U87 spheroids to NK cell-mediated killing $(a, b, c)$. MHT-treated or untreated U87 cells were cultured for 24 hours in serum-free neuronal stem cell medium, following this resting period, IL-2-activated NK cells were added to the culture. At day 7 each culture well was analyzed measuring the area of the spheroids by imageJ software. Representative images per each condition are reported (a). Results are presented as mean \pm SEM of three experiments (b). Cell viability was also evaluated by crystal violet staining (c). U87 cells were gently harvested and incubated in conventional adhesion conditions. After 48 hours, cell cultures were stained with crystal violet followed by extensive washes (to remove non-adherent cells) and lysis of adherent cells. Relative absorbance at $595 \mathrm{~nm}$ was measured with a plate reader. Data are expressed 
as mean \pm SD of three experiments. Chemotaxis of NK cells toward chemokines produced by MHTtreated cells was evaluated using a transwell migration assay $(\mathrm{d})$. Conditioned medium collected from U87 cells untreated or treated with IONCs and MHT were added to the lower chambers of transwell plates. Fresh medium was used as a control. NK cells $\left(1 \times 10^{5}\right)$ were added to the upper chamber and cells were incubated for 18 hours. Cells in the lower chambers were harvested and counted. Data are shown as mean \pm SEM of four independent experiments. Statistical analysis was performed using a one-way ANOVA test $\left({ }^{*} 0.01<\mathrm{p}<0.05 ;{ }^{* *} 0.001<\mathrm{p}<0.01 ;{ }^{* * *} \mathrm{p}<0.001\right.$; n.s. $=$ not significant).

The next experiments were addressed at evaluating the impact of MHT treatment on NK cell cytotoxicity against U87 cells. NK cell cytotoxic activity against tumor cells is mainly exerted via the secretion of cytotoxic granules containing granzymes/perforin, resulting in surface exposure of lysosomal-associated proteins that are typically present on the lipid bilayer surrounding lytic granules, such as CD107a. Therefore, we evaluated the membrane expression of CD107a on NK cells as a marker of cytotoxic degranulation. For this, we co-cultured IL-2-activated NK cells, isolated from two healthy donors, with untreated and MHT-treated U87 spheroids, and quantified tumor cell killing and the levels of CD107a in NK cells (Figure 8). Interestingly, pre-treatment of U87 spheroids with MHT resulted in increased sensitivity to NK cell cytotoxic action $(p<0.001)$ (Figure $\mathbf{8 a}$ and $\mathbf{2 b}$ ) and, in line with this, MHT pre-treated U87 spheroids induced higher levels of NK cell degranulation compared to untreated U87 spheroids (Figure 8c and 8d). The enhanced NK cell reactivity against MHT-pretreated GBM spheroids was also observed in terms of IFN- $\gamma$ production (Figure S7 in the supporting information). -Finally, we demonstrated that the killing of MHT pre-treated U87 GBM spheroids, involves both DNAM-1 and NKp30 receptors, since double blocking treatment with anti-DNAM-1 and anti-NKp30 antibodies caused a strong inhibition of killing and degranulation of IL-2-activated NK cells, compared with single blocking of DNAM-1 or NKp30 ( p < 0.001).
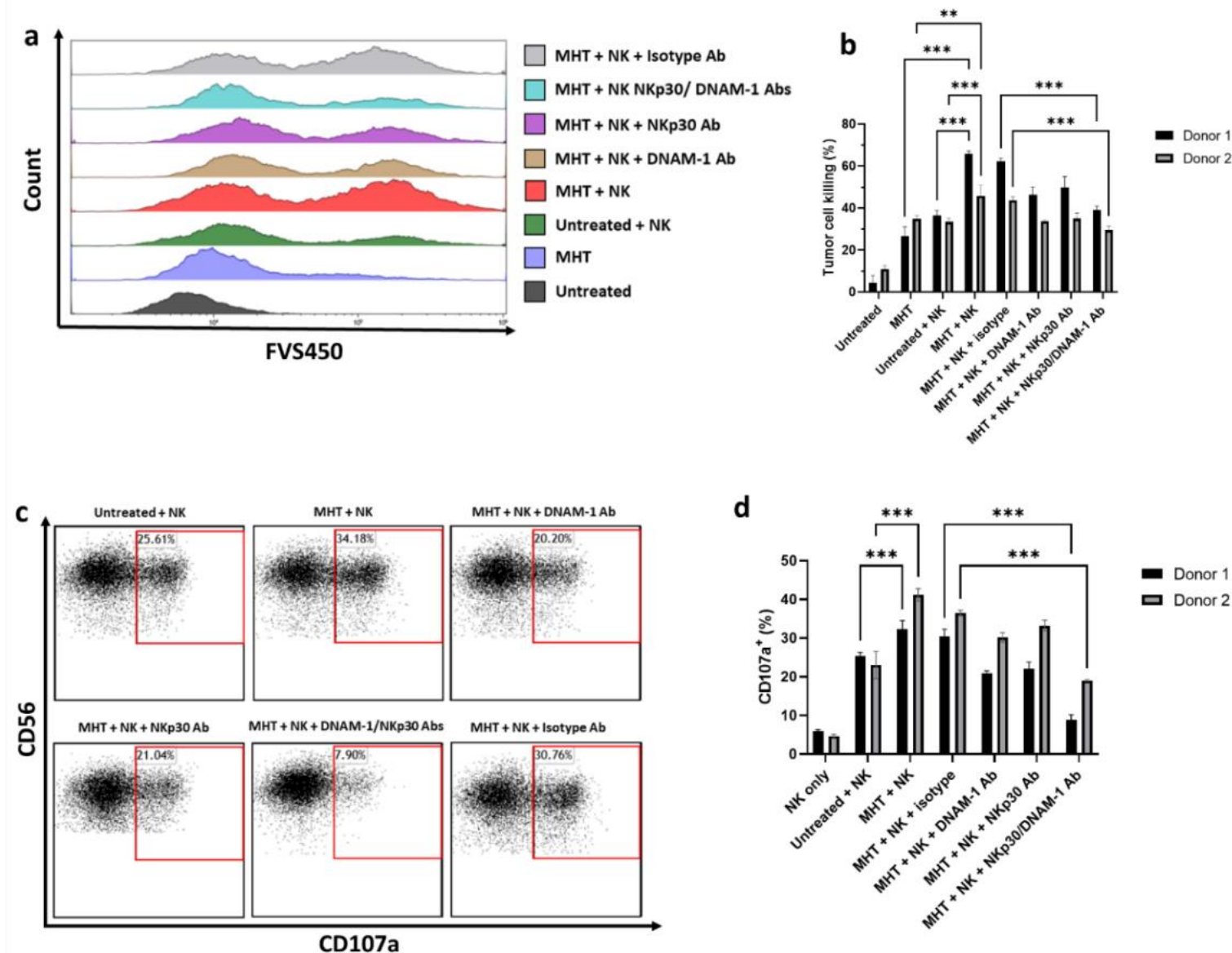
Figure 8. NK cell-mediated killing of U87 cells and degranulation activity. U87 cells were treated with MHT and co-cultured with IL-2-activated NK cells for 4 hours at an E:T ratio of 1:1. Tumor cell killing of U87 cells was assessed by flow cytometry determining viability dye-positive (FSV450+) cells in U87 cells. (a) Data are shown as representative histograms (a) and mean \pm SEM. (b). IL-2activated NK cells were co-incubated with U87 cells for $4 \mathrm{~h}$ at an E:T ratio of 1:1 in the presence of anti-CD107a antibody. Percentage of degranulating CD107a+/CD56+ NK cells was represented as individual plots (c) and mean \pm SEM (d). Results shown are representative of three experiments using NK cells from two healthy donors. Statistical analysis was performed using a one-way ANOVA test $\left({ }^{*} 0.01<\mathrm{p}<0.05 ;{ }^{* *} 0.001<\mathrm{p}<0.01 ;{ }^{* * *} \mathrm{p}<0.001 ;\right.$ n.s. $=$ not significant $)$.

\section{Discussion}

The exploitation of ICD to revert the immunosuppressive TME into a more immunostimulatory one, and trigger an effector antitumor immune response sustained by both innate and adaptive effector cells against stressed/dying cancer cells is emerging as a novel strategy for the treatment of tumors that are classified as immunologically "cold".

This is particular interesting for GBM since recent evidence suggests a predominant role of innate immunity in brain tumor surveillance. Indeed, GBM shows a "non-conventional" immunological profile and unlike other tumors, such as melanoma and breast cancer, NK cells represent the most abundant tumor-infiltrating lymphocytes and up to half of GBM mass is constituted by resident microglia and circulating blood monocytes/macrophages [63,64].

Although MHT-induced anticancer responses have been reported in several cancers, including melanoma, breast cancer, colon carcinoma, little is known regarding MHT as ICD-inducer in GBM $[53,54]$. Our results demonstrate that mild MHT treatment can be exploited as the external activation mechanism to induce ICD and re-modulate the immunogenic profile of U87 GBM cells, thus rendering them more susceptible to the antitumoral action of innate immune effectors, such as macrophages and NK cells. The results of the present study demonstrated that treatment with mild MHT was cytotoxic in U87 cells, with $30 \%$ reduction in cell viability, and that it can re-shape the immunological features of these tumor cells thus facilitating their recognition by innate effectors, including macrophages and NK cells.

MHT treatment, mediated by IONC-GA-PEG, induced the upregulation of several immunogenic molecules (CRT, HSP70, HSP90 and HMGB-1) and downregulated the expression of immunological "breaks" (CD47 and PD-L1) that can promote immune evasion. Differential expression of DAMPs resulted in a faster recognition and phagocytosis of U87 cells by macrophages in vitro. Upon 24 hours of co-culture, macrophages incubated in the presence of U87 cells pre-treated with mild MHT displayed an activated profile with increased surface levels of CD86 and MHC-II.

Once we showed that, in line with other previous studies conducted with other type of cancer cells, MHT can induce ICD in U87 GBM cells, we decided to draw our attention to the potential role that NK cells can play in mediating MHT-triggered immune responses.

First, we showed that treatment with mild MHT induces increased expression of DNAM-1 and NKp30 ligands and downregulation of MHC molecules in U87 human GBM cells, thereby potentially enhancing their susceptibility to NK cell-mediated killing. Consequently, we found that pre-treatment of GBM cells with MHT positively reflected on the functionality of IL-2-activated NK cells in terms of degranulation and IFN- $\gamma$ release.

Importantly, DNAM-1 and NKp30 blockade reduced the lysis of cancer target cells, and together the two blocking antibodies displayed a synergistic effect that abrogate NK cell-mediated killing of U87 cells pre-sensitized by treatment with MHT. This result is consistent with the reduction in NK cell degranulation upon pre-treatment with antiDNAM-1 and NKp30 blocking antibodies of MHT-treated GBM cells. Together, these findings provide clear evidence that mild MHT treatment cannot only induce adaptive immunity by serving as ICD-inducer for the activation of APCs, but can also sensitize U87 GBM cells to NK cell-mediated killing. 
Even if new ablative physical treatments have been recently approved for clinical use in patients with GBM, including tumor treating fields (TTFields) and laser interstitial thermal therapy (LITT), important challenges are still associated with TTFields and LITT, which include the elevated costs associated with these medications and the limited applicability to only certain subtypes of GBMs depending on their size, morphology and localization [67-69]. Moreover, the ability of TTFields and LITT to serve as ICD-inducers still needs to be proven. The latter is particularly true in the case of TTFields which induce cell death mostly via apoptosis, that has been demonstrated to fail at triggering APC activation and, instead, promotes immune tolerance [70]. Furthermore, unlike laser-based treatments, MHT provides a remote activation modality with no tissue-penetration depth problems.

All the above, together with the demonstrated ability to target CSCs makes MHT one of the most valid options as ICD-inducer among the various approved ablative treatments [71].

\section{Conclusions}

In summary, we showed that mild MHT with magnetic nanoparticles can be apply not only as an "in situ vaccination" strategy, but can be also explored as a novel approach to enhance the antitumoral activity of NK cells in GBM. This occurs by re-modulating the immunological profile of target cells via induction of stress-associate molecules and downregulating inhibitory ligands. These results could open new therapeutic possibilities for MHT, providing a rationale for novel combinatorial treatments based on MHT and NK cell therapies, with the potential to ensure simultaneously TIME re-modulation and the generation of a NK cell-sustained effector response. However, at the same time, we also observed slight reduction in the expression of some NKG2D ligands on the surface of U87 cells that were exposed to mild MHT. Thus, future in vitro and in vivo studies are needed to further explore the association of mild MHT treatment with the expression of NK ligands. Thereby, it will be possible to define if this concept can be generalized to GBM or even extended to other kind of tumors. Furthermore, a comprehensive elucidation of the molecular mechanisms behind the effects of mild MHT on the tumor-NK cell interaction could enable a full exploitation of NK cell-based therapeutic potential.

\section{Experimental section}

U87 and THP-1 cell lines were purchased from American Type Culture Collection (ATCC). All chemicals were obtained from Sigma-Aldrich. Annexin-FITC/PI kit was obtained from Miltenyi Biotec. Antibodies for E-cadherin and Vimentin were purchased from Santa Cruz Biotechnology. Antibodies against CRT, HSP70 and HSP90 were obtained from Enzo Life Sciences. Antibodies for CD133, CD47, CD14, HLA-I (ABC), HLAE, HLA-DR, CD11b, CD86 and PD-L1 were purchased from Biolegend. Antibodies against ULBP-1, ULBP-2 and ULBP-3 were purchased from R\&D Systems. Anti-CD16 antibody was obtained from Miltenyi Biotec. Anti-CD107a antibody was purchased from ThermoFisher Scientific. Anti-CD56 antibody was purchased from Beckman Coulter. Fixable viability staining 450 (FVS450) was provided by BD Biosciences. Carboxyfluorescein diacetate succinimidyl ester (CFSE) was purchased from Thermo Fisher Scientific. Antibodies against Nectin-2, PVR, B7H6 were obtained from R\&D Systems. HMGB1 ELISA kit was obtained from Tecan. IFN- $\gamma$ ELISA kit was purchased from Invitrogen.

\section{Synthesis of the IONCS}

The synthesis of the IONCs was conducted according to the procedure reported in the patent [58]. Briefly, to prepare the IONCs a solvothermal method was used and it requires three steps: i) providing a solution comprising oleic acid, hexadecylamine and 1octanol; ii) adding a solution of iron pentacarbonyl and benzaldehyde; iii) transferring the 
reaction mixture to an autoclave vessel, at a filling percentage thereof of $46 \%$ in $\mathrm{V}$; and iv) heating the autoclave to a temperature of $200^{\circ} \mathrm{C}$ for 6 hours.

Synthesis of Gallic Acid PEG (GA-PEG) ligand

The synthesis of GA-GEG was that reported by P. Guardia et al. with some modifications [59]. Briefly, $5 \mathrm{~g}$ Poly(ethylene glycol) (PEG, $3.3 \mathrm{mmol}, \mathrm{Mw}=1500 \mathrm{kDa}$ ) were dissolved in $500 \mathrm{~mL}$ tetrahydrofuran (THF) through sonication for $1 \mathrm{~h}$. $280 \mathrm{mg}$ of Gallic acid (GA, $1.64 \mathrm{mmol}$ ) was dissolved in $10 \mathrm{~mL}$ THF and separately $20 \mathrm{mg}$ dimethyl amino pyridine (DMAP, $0.16 \mathrm{mmol}$ ) were dissolved in $10 \mathrm{~mL}$ THF in a flask under magnetic stirring at room temperature. $1.72 \mathrm{~g} \mathrm{~N} . \mathrm{N}^{\prime}$-Dicyclohexylcarbodiimide (DCC, $8.3 \mathrm{mmol}$ ) dissolved in $20 \mathrm{~mL}$ of THF was drop-wise added within 1 h to the solution of PEG/GA/DMAP under energic magnetic stirring. The mixture was then stirred at room temperature for additional 48 hours and THF and DMAP were removed under reduced pressure (300 mbar). The crude product (GA-PEG) was completely dried and then dissolved in $40 \mathrm{~mL}$ de-ionized water and the $\mathrm{pH}$ adjusted to 2 to precipitate hydrolyzed DCC. After 1h, the solution was filtered with a paper filter in a Buchner funnel and GA-PEG was extracted from the aqueous phase with $200 \mathrm{~mL}$ chloroform. This step was repeated 5 times. After removal of Chloroform at reduced (350 mbar) pressure, GA-PEG was dried in the vacuum stove overnight at $44^{\circ} \mathrm{C}$. GA-PEG was dissolved in $20 \mathrm{~mL}$ of dichloromethane (DCM) and added dropwise in $200 \mathrm{~mL}$ of cold diethyl ether. After this, the precipitate was filtered with a Buchner funnel and dried in the vacuum stove overnight at $40{ }^{\circ} \mathrm{C}$. Prior to determination of the yield, a $0.05 \mathrm{M}$ solution of GA-PEG in chloroform was prepared for further used for the water transfer of the IONCs. The yield was determined through ${ }^{1} \mathrm{H}-\mathrm{NRM}$ and was found to be $11 \%$.

\section{Ligand Exchange and Water Transfer of Cubic Iron Oxide Nanoparticles (IONCs-GA-PEG)}

Briefly, $4 \mathrm{~mL}$ of a chloroform solution of the IONCs $([\mathrm{Fe}]=1 \mathrm{mg} / \mathrm{mL})$ was added to a $10.8 \mathrm{~mL}$ GA-PEG solution $(0.05 \mathrm{M})$ to provide 500 ligand molecules per $\mathrm{nm}^{2}$ of nanoparticle surface. Subsequently, $1.08 \mathrm{~mL}$ of triethylamine (TEA) was added. The solution was stirred in an orbital shaker overnight at $3000 \mathrm{rpm}$. The sample was extracted with $5 \mathrm{~mL}$ of toluene, $10 \mathrm{~mL}$ of Milli-Q water and small amounts of acetone. IONCs spontaneously phase transferred from the organic to the aqueous phase. Any trace of acetone was removed from the aqueous phase by bubbling nitrogen for at least $30 \mathrm{~min}$. The sample was concentrated through rotavaporation and it was further purified by dialysis overnight and at room temperature against Milli-Q water (5 L) to remove the unreacted GA-PEG. Cellulose membrane tubes with a molecular weight cut-off (MWCO) of $50 \mathrm{kDa}$ were chosen for the dyalisis. This step was repeated two times. Finally, the resulting GA-PEG-coated IONC sample was concentrated by ultrafiltration using an Amicon centrifugal filter (MWCO of $100 \mathrm{kDa}$, Merck Millipore) and analyzed by dynamic light scattering (DLS) (Zetasizer Nano ZS90, Malvern) and transmission electron microscopy (TEM).

\section{SAR measurements}

The calorimetric measurements to quantify the specific absorption rate (SAR) value of the IONCs were conducted using a commercially available magnetic nano-heating device (DM100 Series, nanoscale Biomagnetics). The aqueous solution of IONCs was exposed to an alternating magnetic field with amplitudes of 12,16 or $24 \mathrm{kA} / \mathrm{m}$ and frequencies of 100 or $300 \mathrm{kHz}$. SAR values were calculated using the following equation:

$$
\mathrm{SAR}=(\mathrm{C} / \mathrm{m})(\mathrm{dT} / \mathrm{dt}),
$$

where $C$ is the specific heat capacity of the colloid $\left(C_{\text {water }}=4.18 \mathrm{~J} \mathrm{~g}^{-1} \mathrm{C}^{-1}\right), \mathrm{dT} / \mathrm{dt}$ is the initial slope of the time-dependent temperature curve, and $\mathrm{m}$ is mass of magnetic material $(\mathrm{g} / \mathrm{L})$ in the suspension. To calculate the parameter $\mathrm{dT} / \mathrm{dt}$, temperature data points collected within the first 60 seconds were used to obtain the slope of the curve deriving from the linear fitting of these points. 
U87 cells were cultured in Dulbecco's modified Eagle's medium (DMEM; Sigma-Aldrich - high glucose with $4500 \mathrm{mg} / \mathrm{L}$ of glucose) and supplemented with $10 \%$ heat-inactivated fetal bovine serum (FBS; Sigma-Aldrich), $2 \mathrm{mM}$ L-glutamine (Sigma-Aldrich) and $100 \mathrm{U} / \mathrm{mL}$ penicillin/streptomycin (Sigma-Aldrich) at $37^{\circ} \mathrm{C}$ in a humidified $95 \%$ air and $5 \% \mathrm{CO}_{2}$ atmosphere.

U87 spheroids were cultured in serum-free neural stem cell culture medium composed of DMEM/F-12 (Sigma-Aldrich) supplemented with B-27 without vitamin A, 20 $\mathrm{ng} / \mathrm{mL}$ of both epidermal growth factor (EGF; PeproTech), $20 \mathrm{ng} / \mathrm{mL}$ basic fibroblast growth factor (FGF; PeproTech), $5 \mu \mathrm{g} / \mathrm{mL}$ heparin and $1 \%$ penicillin/streptomycin into ultra-low attachment 96-well microplates with flat bottom (Corning).

THP-1 cells were grown in RPMI-1640 (Sigma-Aldrich) supplemented with $10 \%$ heatinactivated FBS, $2 \mathrm{mM}$ L-glutamine, $100 \mathrm{U} / \mathrm{mL}$ penicillin/streptomycin and $50 \mu \mathrm{M} \beta$-mercaptoethanol (Sigma-Aldrich). THP-1 monocytes were differentiated into macrophages by 48 hours incubation with $100 \mathrm{ng} / \mathrm{mL}$ phorbol 12-myristate 13-acetate (PMA, Sigma-Aldrich) followed by 48 hours incubation in RPMI 1640 medium. Differentiation was confirmed by evaluating the expression of CD14 using a BD FACSAria III flow cytometry (BD Biosciences) (Figure S8 in the supporting information).

\section{Cell treatment with $\mathrm{MHT}$}

Magnetic hyperthermia studies on cells were performed at $182 \mathrm{kHz}$ and $16 \mathrm{kA} / \mathrm{m}$ field conditions for exposure to three consecutive cycles of MHT separated by a 5-minute break under AMF of 30 minutes each and at a IONCs concentration of 2 gFe/L. In detail, adherent U87 cells were detached with trypsin and counted using a hemocytometer. Afterwards, $1.5 \times 10^{6}$ cells were resuspended in $150 \mu \mathrm{L}$ of medium containing IONCs within a small glass vial. Then, cells were exposed to AMF. Immediately after treatment, the cells were washed with PBS and resuspended in serum-free neural stem cell culture medium.

\section{Cell viability assay}

To measure cell viability, cells were plated in 96 -well plates $\left(5 \times 10^{3}\right.$ cells per well) and allowed to grow for 24 hours before treatment. Cells were then exposed to different treatments, such as only magnetic field without IONCs (AMF), IONCs and with no exposure to AMF (IONCs) and IONCs and MHT exposure (MHT). After incubation for 24 and 48 hours, cell viability was evaluated by using a CCK-8 assay kit (Abcam) according to the manufacturer's instructions.

\section{Annexin V-FITC/Propidium Iodide assay}

Cell death was determined by using Annexin V-FITC/Propidium Iodide (PI) kit (MACS, Miltenyi Biotech) according to the manufacturer's instructions. Briefly, after treatment, cells were washed with $1 \times$ binding buffer and then $10^{6}$ cells from each group were stained with AnnexV-FITC for $15 \mathrm{~min}$ in the dark, followed by PI staining. The stained cells were analyzed by flow cytometry (BD FACSAria III). The results were expressed as percentage of living (AnnexV-/PI-), primary necrotic (AnnexV-/PI+), apoptotic (Annex $\mathrm{V}^{+} / \mathrm{PI}$ ) and secondary necrotic cells $\left(\right.$ Annex $\left.\mathrm{V}^{+} / \mathrm{PI}^{+}\right)$. Data were analyzed using FCS express 7 software (DeNovo Software).

\section{Flow cytometry analysis of surface markers on U87 cells}

For flow cytometry analysis, U87 cells were collected by using TrypLE Express Enzyme (Gibco) or Gentle Cell Dissociation Reagent (StemCell Technologies). Cells were then transferred into $15 \mathrm{~mL}$ tubes (Sigma-Aldrich) and washed with FACS buffer (ice-cold PBS with $2 \%$ FBS). Then $1 \times 10^{5}$ cells were resuspended in $100 \mu$ of FACS buffer containing antibodies and incubated for $30 \mathrm{~min}$ on ice. All subsequent incubation steps were carried out in the dark. Cells were washed with $1 \mathrm{~mL}$ of FACS buffer and centrifuged at $300 \mathrm{~g}$ for 5 minutes at $4^{\circ} \mathrm{C}$. Then, the cells were resuspended in FACS buffer and analyzed using a 
BD FACSAria III. Unspecific background of individual channels was determined with isotype controls and color compensation was done on single color-stained samples. FACS plots were generated with FCS express 7 software (DeNovo Software).

Cell cycle analysis

Cells were harvested and washed twice with ice-cold $1 \times$ PBS. Then, $1 \times 10^{6}$ cell pellets were resuspended in $4.0 \mathrm{~mL}$ ice-cold $70 \%$ ethanol by adding with a Pasteur pipette on a vortex. After 2-hour incubation at $4^{\circ} \mathrm{C}$, cells were pelleted by centrifugation at $1000 \mathrm{~g}$ for 5 minutes and washed twice with $1 \times$ PBS. Then, cells were resupended with $300 \mu \mathrm{L}$ of DAPI $(1 \mu \mathrm{g} / \mathrm{mL}$, Sigma-Aldrich)/ Triton X-100 (0.1\%, Sigma-Aldrich) solution and incubated 30 minutes at room temperature protected from the light. The cells were analyzed for DNA content by flow cytometry (BD FACSAria III). The relative proportions of cells in G0-G1 phase $(2 n), S$ phase $(>2 n$ but $<4 n)$, and G2/M phase $(4 n)$ of the cell cycle were determined using FCS express 7 software (DeNovo Software).

\section{Transwell migration assay}

Transwell migration assay of U87 cells was performed using a 24-well transwell plate containing inserts with polycarbonate membrane with pores of $8 \mu \mathrm{m}$ (Corning). $1 \times 10^{5}$ cells resuspended in $100 \mu \mathrm{L}$ of serum-free medium were placed in the upper insert and the outer chamber was filled with $600 \mu \mathrm{L}$ of medium containing $10 \%$ FBS. After incubation for 18 hours, the inserts were transferred to a new plate containing pre-warmed $\left(37^{\circ} \mathrm{C}\right)$ trypsin-EDTA. Medium with $10 \%$ FBS was added to inactivate trypsin. Cells that had migrated through the pores into the lower chamber were detected by measuring their cellular ATP content using a CellTiter-Glo 2.0 reagent. The luminescence was measure at 548 $\mathrm{nm}$ using a microplate reader (Tecan Spark).

Chemotaxis of NK cells was tested by a transwell assay. Briefly, lower chambers of 24-well transwell plates ( $3.0 \mu \mathrm{m}$ pore size, Corning) were filled with $600 \mu \mathrm{L}$ of conditioned medium collected from untreated cells or cells exposed to different treatments. $600 \mu \mathrm{L}$ of fresh medium were used as a control. Approximately $1 \times 10^{5}$ of NK cells were added in $100 \mu \mathrm{L}$ of serum-free medium (RPMI containing $25 \mathrm{mM}$ HEPES and $0.5 \%$ BSA) in the upper chamber, and cells were incubated for 18 hours. Cells were harvested by centrifugation (300g for 5 minutes at $4^{\circ} \mathrm{C}$ ), resuspended in $100 \mu \mathrm{l}$ PBS, stained with trypan blue and counted using a hemocytometer.

\section{Colony formation assay}

Immediately after treatment, cells were grown under adherent conventional conditions with complete medium into 6-well plates (500 cells per well) for 14 days. Then, the cells were washed with $1 \times$ Phosphate-buffered saline (PBS, Sigma-Aldrich), fixed with icecold methanol for 10 minutes at room temperature and subsequently stained with $0.5 \%$ crystal violet (Sigma-Aldrich) for 15 minutes. After washing with water, the stained cells were photographed, and cell growth was quantified by dissolving crystal violet in SDS $(2 \%)$ and measuring the absorbance at $595 \mathrm{~nm}$ (Multiskan, Thermo Fisher Scientific).

\section{In vitro phagocytosis, killing and activation of THP-1-derived macrophages}

Dissociated cancer cells were washed twice and resuspended at $1 \times 10^{6}$ cells $/ \mathrm{mL}$ in 1 $\mathrm{mL}$ of $1 \times \mathrm{PBS}$ containing CFSE $(5 \mu \mathrm{M})$. After incubation of 10 minutes in a $37^{\circ} \mathrm{C}$ water bath, the cells were washed with ice-cold $1 \times$ PBS to remove the excess of CFSE.

Stained cells were resuspended in serum-free DMEM at a concentration of $5 \times 10^{4}$ cells $/ \mathrm{mL}$ and transferred into a 24-well plate containing THP-1-derived macrophages $\left(5 \times 10^{4}\right.$ cells $\left./ \mathrm{mL}\right)$, prepared as previously described, for a final Effector: Target ratio (E:T ratio) of 1:1. Cells were co-cultured for two hours, then harvested, stained with anti-human CD11b for 30 minutes at $4^{\circ} \mathrm{C}$ and washed twice with $2 \%$ FBS in $1 \times$ PBS.

Phagocytosis was determined by flow cytometry and data analysis performed using FCS express 7 software (DeNovo Software). The phagocytic index was calculated as the

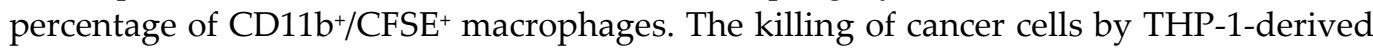


macrophages was evaluated by crystal violet staining following 48 hours of incubation and quantified by measuring the absorbance at $595 \mathrm{~nm}$ with a plate reader (Multiskan, Thermo Fisher Scientific). For stimulation with TLR-agonist a Class C CpG-ODN (ODN 2395) was used at a concentration of $1 \mu \mathrm{g} / \mathrm{mL}$. The killing was calculated using the following formula: killing $(\%)=[($ Absorbance $($ treated tumor cells + Macrophages) - Absorbance (Macrophages) $) /$ (Absorbance (Treated tumor cells + Macrophages) - Absorbance(Macrophages) $)] \times 100$.

The expression of cell surface activation markers on THP-1 cells was analyzed by flow cytometry. THP- 1 macrophages were detached from culture flasks by gentle scraping and incubated for 30 minutes at $4^{\circ} \mathrm{C}$ in PBS with $2 \%$ FBS containing anti-CD11b, anti.CD86, anti-HLA-DR antibodies. The expression of CD86 and HLA-DR on CD11b cells was analyzed by BD FACSAria III (BD Biosciences), and data were processed by FCS express 7 software (DeNovo Software).

\section{HMGB-1 ELISA}

After exposure to different treatments, U87 cells were seeded in 24-well plates and incubated for 24 hours. Supernatants were collected for high mobility group box 1 (HMGB-1) detection by ELISA kit (Tecan), according to the manufacturer's instructions.

\section{NK cell preparation}

NK cells were isolated from peripheral blood by Ficoll-Paque density gradient to obtain Peripheral Blood Mononuclear Cells (PBMCs) followed by purification using an NK cell isolation kit (RosetteSep kit, StemCell Biotechnologies). After isolation, NK cells were cultured in round-bottom 96-well plates in $200 \mu$ l of complete RPMI 1640 medium (SigmaAldrich) supplemented with 10\% fetal calf serum (FCS), $2 \mathrm{mM}$ L-glutamine, $100 \mathrm{IU} / \mathrm{mL}$ rhIL-2 and $100 \mathrm{U} / \mathrm{mL}$ penicillin/streptomycin, and containing $10^{5}$ irradiated PBMC and $5 \times 10^{3} 721.221$ lymphoblastoid cell line transfected with HLA-G. CD3-CD56+clones were obtained by culturing highly purified $\mathrm{CD}^{-} \mathrm{CD} 19^{-} \mathrm{CD} 14^{-} \mathrm{NK}$ cells under limiting dilution conditions as previously reported[72].

\section{NK cell functional assays}

Untreated and treated cells were cultured in serum-free neural stem cell culture medium at $5 \times 10^{3}$ cells/well in flat-bottom ultra-low attachment 96-well plates (Corning). At 24 hours post-treatment, IL-2-activated NK cells $(100 \mathrm{U} / \mathrm{mL})$ were added to the U87 cells at $2.5 \times 10^{4}$ cells $/ \mathrm{mL}$. Generation of spheroids was monitored till day 7 and spheroid area was analyzed in each culture well by acquiring images with a default microscope with $4 \times$ objective and analyzing them with imageJ software. The supernatants were collected to measure the release of IFN- $\gamma$ by ELISA kit according to manufacturer's instructions (Biolegend).

Crystal violet cytotoxicity assay was also performed to determine the killing activity of NK cells. Briefly, spheroids at day 5, alone or co-cultured with NK cells, were transferred in conventional adherent plates and after 48 hours were stained with crystal violet as previously described. After extensive washing, adherent cells were solubilized and the amount of crystal violet proportional to the number of living cells was estimated with a plate reader (Multiskan, Thermo Fisher Scientific) measuring the absorbance at $595 \mathrm{~nm}$.

Degranulation and viability were assessed by flow cytometry on NK cells and U87 target cells, respectively. Briefly, U87 cells exposed or not to treatment with MHT were incubated 24 hours in serum-free neural stem cell culture medium. Then, U87 cells were co-culture with IL-2-activated NK cells $(100 \mathrm{U} / \mathrm{mL})$ at an E:T ratio of 1:1 $\left(1 \times 10^{5}\right.$ cells: $1 \times 10^{5}$ cells) in the presence of eFluor660-conjugated anti-CD107a. Before co-culture with target cells, NK cells were either left untreated or blocked for $30 \mathrm{~min}$ with anti-DNAM-1 antibody $(10 \mu \mathrm{g} / \mathrm{mL}$, Miltenyi Biotec), or anti-NKp30 antibody (10 $\mu \mathrm{g} / \mathrm{ml}$, Miltenyi Biotec). After 1 hour of culture, monensin (BD golgi stop, BD Biosciences) was added and cells were cultured for a further 3 hours before staining for CD56 and viability with Fixable Viability Stain 450 (FVS450, BD Horizon). 
Statistical analysis

Graphpad prismTM 7.0 software was used for analyzing and graphing the data. Statistical significance was determined using unpaired student's $t$ test or one-way ANOVA. Differences were considered statistically significant if $\mathrm{p}<0.05\left({ }^{*} \mathrm{p}<0.05 ;{ }^{* *} \mathrm{p}<0.01\right.$; ${ }^{* * *} \mathrm{p}<$ 0.001 ; and n.s., not significant). Data were expressed as mean $\pm \mathrm{SD}$ or mean $\pm \mathrm{SEM}$.

\section{Author contribution:}

Conceptualization, S.P.; data curation, S.P., F.V; formal analysis, S.P.; investigation, S.P. F.V., A.P., J.L.C.F., G.M.R.R., H.G., N.S. funding acquisition, S.P., T.P.; supervision, T.P., A.P.; software, S.P; visualization, S.P.; drafted, S.P; reviewed and edited, S.P., H.G., N.S., T.P., A.P. All authors have read and agreed to the published version of the manuscript.

\section{Funding:}

This work was partially supported by the Marie Skłodowska-Curie Actions (MSCA) grant within the European Union's Horizon 2020 program (Grant No. 843838 to SP) and partially by AIRC Foundation (AIRC IG-14527 to T.P).

\section{Conflicts of Interest:}

T.P. and H.G. are inventors on patent number WO2020222133A1. All the other authors declare no conflict of interest.

\section{Data Availability Statement:}

The data presented in this study are available within the article and supplementary materials.

\section{References}

1. Ostrom, Q.T.; Gittleman, H.; Fulop, J.; Liu, M.; Blanda, R.; Kromer, C.; Wolinsky, Y.; Kruchko, C.; Barnholtz-Sloan, J.S. CBTRUS Statistical Report: Primary Brain and Central Nervous System Tumors Diagnosed in the United States in 2008-2012. Neuro-Oncology 2015, 17, iv1-iv62, doi:10.1093/neuonc/nov189.

2. Fernandes, C.; Costa, A.; Osorio, L.; Lago, R.C.; Linhares, P.; Carvalho, B.; Caeiro, C. Current Standards of Care in Glioblastoma. Therapy; 2017, Chapter 11, doi:15586/codon.glioblastoma.2017.ch11.

3. Dobosz, P.; Dzieciątkowski, T. The Intriguing History of Cancer Immunotherapy. Frontiers in Immunology 2019, 10, 2965, Doi:10.3389/fimmu.2019.02965.

Waldman, A.D.; Fritz, J.M.; Lenardo, M.J. A Guide to Cancer Immunotherapy: From T Cell Basic Science to Clinical Practice. Nature Reviews Immunology 2020, 20, 651-668, doi:10.1038/s41577-020-0306-5.

5. $\quad$ Barone, A.; Hazarika, M.; Theoret, M.R.; Mishra-Kalyani, P.; Chen, H.; He, K.; Sridhara, R.; Subramaniam, S.; Pfuma, E.; Wang, Y.; et al. FDA Approval Summary: Pembrolizumab for the Treatment of Patients with Unresectable or Metastatic Melanoma. Clinical Cancer Research 2017, 23, 5661-5665, doi:10.1158/1078-0432.CCR-16-0664.

6. Huang, Z.; Su, W.; Lu, T.; Wang, Y.; Dong, Y.; Qin, Y.; Liu, D.; Sun, L.; Jiao, W. First-Line Immune-Checkpoint Inhibitors in Non-Small Cell Lung Cancer: Current Landscape and Future Progress. Frontiers in Pharmacology 2020, 11, 1-16, doi:10.3389/fphar.2020.578091.

7. Pusztai, L.; Karn, T.; Safonov, A.; Abu-Khalaf, M.M.; Bianchini, G. New Strategies in Breast Cancer: Immunotherapy. Clinical Cancer Research 2016, 22, 2105-2110, doi:10.1158/1078-0432.CCR-15-1315.

8. Johdi, N.A.; Sukor, N.F. Colorectal Cancer Immunotherapy: Options and Strategies. Frontiers in Immunology 2020, 11, 1-18, doi:10.3389/fimmu.2020.01624.

9. Deleuze, A.; Saout, J.; Dugay, F.; Peyronnet, B.; Mathieu, R.; Verhoest, G.; Bensalah, K.; Crouzet, L.; Laguerre, B.; Belaud-Rotureau, M.A.; et al. Immunotherapy in Renal Cell Carcinoma: The Future Is Now. International Journal of Molecular Sciences 2020, 21, 2532, doi:10.3390/ijms21072532.

10. McGranahan, T.; Therkelsen, K.E.; Ahmad, S.; Nagpal, S. Current State of Immunotherapy for Treatment of Glioblastoma. Current Treatment Options in Oncology 2019, 20, 24, doi:10.1007/s11864-019-0619-4.

11. DeCordova, S.; Shastri, A.; Tsolaki, A.G.; Yasmin, H.; Klein, L.; Singh, S.K.; Kishore, U. Molecular Heterogeneity and Immunosuppressive Microenvironment in Glioblastoma. Frontiers in Immunology 2020, 11, 1-18, doi:10.3389/fimmu.2020.01402.

12. Antunes, A.R.P.; Scheyltjens, I.; Duerinck, J.; Neyns, B.; Movahedi, K.; Van Ginderachter, J.A. Understanding the Glioblastoma Immune Microenvironment as Basis for the Development of New Immunotherapeutic Strategies. eLife 2020, 9, 1-16, doi:10.7554/eLife.52176.

13. Brown, N.F.; Carter, T.J.; Ottaviani, D.; Mulholland, P. Harnessing the Immune System in Glioblastoma. British Journal of Cancer 2018, 119, 1171-1181, doi:10.1038/s41416-018-0258-8. 
14. Ricklefs, F.L.; Alayo, Q.; Krenzlin, H.; Mahmoud, A.B.; Speranza, M.C.; Nakashima, H.; Hayes, J.L.; Lee, K.; Balaj, L.; Passaro, C.; et al. Immune Evasion Mediated by PD-L1 on Glioblastoma-Derived Extracellular Vesicles. Science Advances 2018, 4, doi:10.1126/sciadv.aar2766.

15. Angell, H.K.; Bruni, D.; Carl Barrett, J.; Herbst, R.; Galon, J. The Immunoscore: Colon Cancer and beyond a C. Clinical Cancer Research 2020, 26, 332-339, doi:10.1158/1078-0432.CCR-18-1851.

16. Galon, J.; Bruni, D. Approaches to Treat Immune Hot, Altered and Cold Tumours with Combination Immunotherapies. Nature Reviews Drug Discovery 2019, 18, 197-218, doi:10.1038/s41573-018-0007-y.

17. Bruni, D.; Angell, H.K.; Galon, J. The Immune Contexture and Immunoscore in Cancer Prognosis and Therapeutic Efficacy. Nature Reviews Cancer 2020, 20, 662-680, doi:10.1038/s41568-020-0285-7.

18. Rothlin, C. V.; Ghosh, S. Lifting the Innate Immune Barriers to Antitumor Immunity. Journal for ImmunoTherapy of Cancer 2020, 8, 1-15, doi:10.1136/jitc-2020-000695.

19. Corrales, L.; Matson, V.; Flood, B.; Spranger, S.; Gajewski, T.F. Innate Immune Signaling and Regulation in Cancer Immunotherapy. Cell Research 2017, 27, 96-108, doi:10.1038/cr.2016.149.

20. Moynihan, K.D.; Irvine, D.J. Roles for Innate Immunity in Combination Immunotherapies. Cancer Research 2017, 77, 5215-5221, doi:10.1158/0008-5472.CAN-17-1340.

21. Shi, Y.; Fan, X.; Deng, H.; Brezski, R.J.; Rycyzyn, M.; Jordan, R.E.; Strohl, W.R.; Zou, Q.; Zhang, N.; An, Z. Trastuzumab Triggers Phagocytic Killing of High HER2 Cancer Cells In Vitro and In Vivo by Interaction with Fc $\gamma$ Receptors on Macrophages. The Journal of Immunology 2015, $194,4379-4386$.

Woan, K. V.; Miller, J.S. Harnessing Natural Killer Cell Antitumor Immunity: From the Bench to Bedside. Cancer Immunology Research 2019, 7, 1742-1747, doi:10.1158/2326-6066.CIR-19-0404.

23. Kmiecik, J.; Zimmer, J.; Chekenya, M. Natural Killer Cells in Intracranial Neoplasms: Presence and Therapeutic Efficacy against Brain Tumours. Journal of Neuro-Oncology 2014, 116, 1-9, doi:10.1007/s11060-013-1265-5. notherapy. Clinical Cancer Research 2019, 25, 4202-4210, doi:10.1158/1078-0432.CCR-18-1627.

Castriconi, R.; Daga, A.; Dondero, A.; Zona, G.; Poliani, P.L.; Melotti, A.; Griffero, F.; Marubbi, D.; Spaziante, R.; Bellora, F.; et al. NK Cells Recognize and Kill Human Glioblastoma Cells with Stem Cell-Like Properties. The Journal of Immunology 2009, 182, 3530-3539, doi:10.4049/jimmunol.0802845.

27. Oh, S.J.; Yang, J.I.; Kim, O.; Ahn, E.J.; Kang, W.D.; Lee, J.H.; Moon, K.S.; Lee, K.H.; Cho, D. Human U87 Glioblastoma Cells with Stemness Features Display Enhanced Sensitivity to Natural Killer Cell Cytotoxicity through Altered Expression of NKG2D Ligand. Cancer Cell International 2017, 17, 1-9, doi:10.1186/s12935-017-0397-7.

Tanaka, Y.; Nakazawa, T.; Nakamura, M.; Nishimura, F.; Matsuda, R.; Omoto, K.; Shida, Y.; Murakami, T.; Nakagawa, I.; Motoyama, Y.; et al. Ex Vivo-Expanded Highly Purified Natural Killer Cells in Combination with Temozolomide Induce Antitumor Effects in Human Glioblastoma Cells in Vitro. PLoS ONE 2019, 14, 1-144, doi:10.1371/journal.pone.0212455.

29. Cooper, M.A. Natural Killer Cells Might Adapt Their Inhibitory Receptors for Memory. Proceedings of the National Academy of Sciences of the United States of America 2018, 115, 11357-11359, doi:10.1073/pnas.1815756115.

30. Stary, V.; Stary, G. NK Cell-Mediated Recall Responses: Memory-Like, Adaptive, or Antigen-Specific? Frontiers in Cellular and Infection Microbiology 2020, 10, 1-7, doi:10.3389/fcimb.2020.00208.

31. Nikzad, R.; Angelo, L.S.; Aviles-Padilla, K.; Le, D.T.; Singh, V.K.; Bimler, L.; Vukmanovic-Stejic, M.; Vendrame, E.; Ranganath, T.; Simpson, L.; et al. Human Natural Killer Cells Mediate Adaptive Immunity to Viral Antigens. Science Immunology 2019, 4, doi:10.1126/sciimmunol.aat8116.

32. Romee, R.; Rosario, M.; Berrien-Elliott, M.M.; Wagner, J.A.; Jewell, B.A.; Schappe, T.; Leong, J.W.; Abdel-Latif, S.; Schneider, S.E.; Willey, S.; et al. Cytokine-Induced Memory-like Natural Killer Cells Exhibit Enhanced Responses against Myeloid Leukemia. Science Translational Medicine 2016, 8, doi:10.1126/scitranslmed.aaf2341.

33. Myers, J.A.; Miller, J.S. Exploring the NK Cell Platform for Cancer Immunotherapy. Nature Reviews Clinical Oncology 2021, 18, 85-100, doi:10.1038/s41571-020-0426-7. 
34. Kurdi, A.T.; Glavey, S. V.; Bezman, N.A.; Jhatakia, A.; Guerriero, J.L.; Manier, S.; Moschetta, M.; Mishima, Y.; Roccaro, A.; Detappe, A.; et al. Antibody-Dependent Cellular Phagocytosis by Macrophages Is a Novel Mechanism of Action of Elotuzumab. Molecular Cancer Therapeutics 2018, 17, 1454-1463, doi:10.1158/1535-7163.MCT-17-0998.

35. Seidel, U.J.E.; Schlegel, P.; Lang, P. Natural Killer Cell Mediated Antibody-Dependent Cellular Cytotoxicity in Tumor Immunotherapy with Therapeutic Antibodies. Frontiers in Immunology 2013, 4, 1-8, doi:10.3389/fimmu.2013.00076.

36. Sampson, J.H.; Gunn, M.D.; Fecci, P.E.; Ashley, D.M. Brain Immunology and Immunotherapy in Brain Tumours. Nature Reviews Cancer 2020, 20, 12-25, doi:10.1038/s41568-019-0224-7.

37. Krysko, D. V.; Garg, A.D.; Kaczmarek, A.; Krysko, O.; Agostinis, P.; Vandenabeele, P. Immunogenic Cell Death and DAMPs in Cancer Therapy. Nature Reviews Cancer 2012, 12, 860-875, doi:10.1038/nrc3380.

38. Fucikova, J.; Kepp, O.; Kasikova, L.; Petroni, G.; Yamazaki, T.; Liu, P.; Zhao, L.; Spisek, R.; Kroemer, G.; Galluzzi, L. Detection of Immunogenic Cell Death and Its Relevance for Cancer Therapy. Cell Death and Disease 2020, 11, doi:10.1038/s41419-020-03221-2.

39. Calderwood, S.K.; Gong, J.; Murshid, A. Extracellular HSPs: The Complicated Roles of Extracellular HSPs in Immunity. Frontiers in Immunology 2016, 7, 159, doi:10.3389/fimmu.2016.00159.

40. Garg, A.D.; Vandenberk, L.; Koks, C.; Verschuere, T.; Boon, L.; Van Gool, S.W.; Agostinis, P. Dendritic Cell Vaccines Based on Immunogenic Cell Death Elicit Danger Signals and T Cell-Driven Rejection of High-Grade Glioma. Science Translational Medicine 2016, 8, doi:10.1126/scitranslmed.aae0105.

41. Humeau, J.; Sauvat, A.; Cerrato, G.; Xie, W.; Loos, F.; Iannantuoni, F.; Bezu, L.; Lévesque, S.; Paillet, J.; Pol, J.; et al. Inhibition of Transcription by Dactinomycin Reveals a New Characteristic of Immunogenic Cell Stress. EMBO Molecular Medicine 2020, 12, 1-22, doi:10.15252/emmm.201911622.

42. Wang-Bishop, L.; Wehbe, M.; Shae, D.; James, J.; Hacker, B.C.; Garland, K.; Chistov, P.P.; Rafat, M.; Balko, J.M.; Wilson, J.T. Potent STING Activation Stimulates Immunogenic Cell Death to Enhance Antitumor Immunity in Neuroblastoma. Journal for ImmunoTherapy of Cancer 2020, 8, 1-17, doi:10.1136/jitc-2019-000282.

43. Zhao, X.; Yang, K.; Zhao, R.; Ji, T.; Wang, X.; Yang, X.; Zhang, Y.; Cheng, K.; Liu, S.; Hao, J.; et al. Inducing Enhanced Immunogenic Cell Death with Nanocarrier-Based Drug Delivery Systems for Pancreatic Cancer Therapy. Biomaterials 2016, 102, 187-197, doi:10.1016/j.biomaterials.2016.06.032.

44. Tesniere, A.; Schlemmer, F.; Boige, V.; Kepp, O.; Martins, I.; Ghiringhelli, F.; Aymeric, L.; Michaud, M.; Apetoh, L.; Barault, L.; et al. Immunogenic Death of Colon Cancer Cells Treated with Oxaliplatin. Oncogene 2010, 29, 482-491, doi:10.1038/onc.2009.356. a Tumor-Specific Immune Response. Cancer Research 2011, 71, 4821-4833, doi:10.1158/0008-5472.CAN-11-0950.

46. Wan, C.; Sun, Y.; Tian, Y.; Lu, L.; Dai, X.; Meng, J.; Huang, J.; He, Q.; Wu, B.; Zhang, Z.; et al. Irradiated Tumor Cell-Derived Microparticles Mediate Tumor Eradication via Cell Killing and Immune Reprogramming. Science Advances 2020, 6, 1-15, doi:10.1126/sciadv.aay9789.

47. Adkins, I.; Sadilkova, L.; Hradilova, N.; Tomala, J.; Kovar, M.; Spisek, R. Severe, but Not Mild Heat-Shock Treatment Induces Immunogenic Cell Death in Cancer Cells. OncoImmunology 2017, 6, 1-13, doi:10.1080/2162402X.2017.1311433.

48. Liu, X.; Zheng, J.; Sun, W.; Zhao, X.; Li, Y.; Gong, N.; Wang, Y.; Ma, X.; Zhang, T.; Zhao, L.Y.; et al. Ferrimagnetic Vortex Nanoring-Mediated Mild Magnetic Hyperthermia Imparts Potent Immunological Effect for Treating Cancer Metastasis. ACS Nano 2019, 13, 8811-8825, doi:10.1021/acsnano.9b01979.

49. Guevara, M.L.; Persano, F.; Persano, S. Nano-Immunotherapy: Overcoming Tumour Immune Evasion. Seminars in Cancer Biology 2021, 69 , 238-248, doi:10.1016/j.semcancer.2019.11.010.

50. Persano, S.; Das, P.; Pellegrino, T. Magnetic Nanostructures as Emerging Therapeutic Tools to Boost Anti-tumour Immunity. Cancers 2021, 13, doi.org/10.3390/cancers13112735.

51. Mukherjee, S.; Liang, L.; Veiseh, O. Recent Advancements of Magnetic Nanomaterials in Cancer Therapy. Pharmaceutics 2020, 12, doi:10.3390/pharmaceutics12020147.

52. Mahmoudi, K.; Bouras, A.; Bozec, D.; Ivkov, R.; Hadjipanayis, C. Magnetic Hyperthermia Therapy for the Treatment of Glioblastoma: A Review of the Therapy's History, Efficacy and Application in Humans. International Journal of Hyperthermia 2018, 34, 1316-1328, doi:10.1080/02656736.2018.1430867.

53. Thiesen, B.; Jordan, A. Clinical Applications of Magnetic Nanoparticles for Hyperthermia. International Journal of Hyperthermia 2008, 24, 467474, doi:10.1080/02656730802104757. 
54. Wang, Z.; Zhang, F.; Shao, D.; Chang, Z.; Wang, L.; Hu, H.; Zheng, X.; Li, X.; Chen, F.; Tu, Z.; et al. Janus Nanobullets Combine Photodynamic Therapy and Magnetic Hyperthermia to Potentiate Synergetic Anti-Metastatic Immunotherapy. Advanced Science 2019, 6, doi:10.1002/advs.201901690.

55. Chao, Y.; Chen, G.; Liang, C.; Xu, J.; Dong, Z.; Han, X.; Wang, C.; Liu, Z. Iron Nanoparticles for Low-Power Local Magnetic Hyperthermia in Combination with Immune Checkpoint Blockade for Systemic Antitumor Therapy. Nano Letters 2019, 19, 4287-4296, doi:10.1021/acs.nanolett.9b00579.

56. Toraya-Brown, S.; Sheen, M.R.; Zhang, P.; Chen, L.; Baird, J.R.; Demidenko, E.; Turk, M.J.; Hoopes, P.J.; Conejo-Garcia, J.R.; Fiering, S. Local Hyperthermia Treatment of Tumors Induces CD8+ T Cell-Mediated Resistance against Distal and Secondary Tumors. Handbook of Immunological Properties of Engineered Nanomaterials: Second Edition 2016, 3, 309-334, doi:10.1142/9677.

57. Dayanc, B.E.; Beachy, S.H.; Ostberg, J.R.; Repasky, E.A. Dissecting the Role of Hyperthermia in Natural Killer Cell Mediated Anti-Tumor Responses. International Journal of Hyperthermia 2008, 24, 41-56, doi:10.1080/02656730701858297.

58. Muñoz, N.M.; Dupuis, C.; Williams, M.; Dixon, K.; McWatters, A.; Avritscher, R.; Bouchard, R.; Kaseb, A.; Schachtschneider, K.M.; Rao, A.; Sheth, R.A. Molecularly targeted photothermal ablation improves tumor specificity and immune modulation in a rat model of hepatocellular carcinoma. Communications Biology 2020, 3, 783, doi: 10.1038/s42003-020-01522-y.

59. Lee, S.; Son, B.; Park, G.; Kim, H.; Kang, H.; Jeon, J.; Youn, H.; Youn, B. Immunogenic Effect of Hyperthermia on Enhancing Radiotherapeutic Efficacy. International Journal of Molecular Sciences 2018, 19, 1-21, doi:10.3390/ijms19092795.

60. T. Pellegrino et al. Method for the gram-scale preparation of cubic ferrite nanocrystals for biomedical applications. WO2020222133A1, 5 November 2020.

61. Guardia, P.; Riedinger, A.; Nitti, S.; Pugliese, G.; Marras, S.; Genovese, A.; Materia, M.E.; Lefevre, C.; Manna, L.; Pellegrino, T. One Pot Synthesis of Monodisperse Water Soluble Iron Oxide Nanocrystals with High Values of the Specific Absorption Rate. Journal of Materials Chemistry B 2014, 2, 4426-4434, doi:10.1039/c4tb00061g.

62. Demaria, O.; Cornen, S.; Daëron, M.; Morel, Y.; Medzhitov, R.; Vivier, E. Harnessing Innate Immunity in Cancer Therapy. Nature 2019, 574, 45-56, doi:10.1038/s41586-019-1593-5.

63. Grauer, O.; Jaber, M.; Hess, K.; Weckesser, M.; Schwindt, W.; Maring, S.; Wölfer, J.; Stummer, W. Combined Intracavitary Thermotherapy with Iron Oxide Nanoparticles and Radiotherapy as Local Treatment Modality in Recurrent Glioblastoma Patients. Journal of Neuro-Oncology 2019, 141, 83-94, doi:10.1007/s11060-018-03005-x.

64. Sachet, M.; Liang, Y.Y.; Oehler, R. The Immune Response to Secondary Necrotic Cells. Apoptosis 2017, 22, 1189-1204, doi:10.1007/s10495-017$1413-\mathrm{z}$.

65. Dong, W.; Wu, X.; Ma, S.; Wang, Y.; Nalin, A.P.; Zhu, Z.; Zhang, J.; Benson, D.M.; He, K.; Caligiuri, M.A.; et al. The Mechanism of Anti-PdL1 Antibody Efficacy against Pd-L1-Negative Tumors Identifies Nk Cells Expressing Pd-L1 as a Cytolytic Effector. Cancer Discovery 2019, 9, 1422-1437, doi:10.1158/2159-8290.CD-18-1259.

66. Juneja, V.R.; McGuire, K.A.; Manguso, R.T.; LaFleur, M.W.; Collins, N.; Nicholas Haining, W.; Freeman, G.J.; Sharpe, A.H. PD-L1 on Tumor Cells Is Sufficient for Immune Evasion in Immunogenic Tumors and Inhibits CD8 T Cell Cytotoxicity. Journal of Experimental Medicine 2017, 214, 895-904, doi:10.1084/jem.20160801.

67. Hottinger, A.F.; Pacheco, P.; Stupp, R. Tumor treating fields: a novel treatment modality and its use in brain tumors. Neuro Oncology 2016, 18, 1338-1349, doi:10.1093/neuonc/now182.

68. Lok, E.; San, P.; Hua, V.; Phung, M.; Wong, E.T. Analysis of physical characteristics of Tumor Treating Fields for human glioblastoma. Cancer Medicine 2017, 6, 1286-1300, doi:10.1002/cam4.1095.

69. Maraka, S.; Asmaro, K.; Walbert, T.; Lee, I. Cerebral edema induced by laser interstitial thermal therapy and radiotherapy in close succession in patients with brain tumor. Lasers in Surgery and Medicine 2018, 50, 917-923, doi:10.1002/1sm.22946.

70. Aaes, T.L.; Verschuere, H.; Kaczmarek, A.; Heyndrickx, L.; Wiernicki, B.; Delrue, I.; De Craene, B.; Taminau, J.; Delvaeye, T.; Bertrand, M.J.M.; Declercq, W.; Berx, G.; Krysko, D.V.; Adjemian, S.; Vandenabeele, P. Immunodominant AH1 Antigen-Deficient Necroptotic, but Not Apoptotic, Murine Cancer Cells Induce Antitumor Protection. Journal of Immunology 2020, 204, 775-787, doi: 10.4049/jimmunol.1900072.

71. Sadhukha, T.; Niu, L.; Wiedmann, T.S.; Panyam, J. Effective elimination of cancer stem cells by magnetic hyperthermia. Molecular Pharmaceutics 2013, 10, 1432-1441, doi: 10.1021/mp400015b. 